\title{
On the Auslander-Reiten valued quiver of right peak rings
}

by

Bogumila Klemp and Daniel Simson (Toruń)

Abstract. Let $R$ be a right peak artinian ring (1.1). We prove that under suitable assumptions (3.7) on $R$ there exists a preprojective component of the Auslander-Reiten valued quiver $\Gamma_{\mathrm{sp}}(R)$ of the category $\bmod _{\mathrm{sp}}(R)$ of finitely generated socle projective. $R$-modules. If $R$ admits a splitting poset decomposition (5.0) a splitting structure of the category $\bmod _{\mathrm{sp}}(R)$ is described in (5.12).

1. Introduction. We recall from [28] that a semiperfect ring $R$ is a right peak ring if $R$ is a generalized matrix ring of the form

$$
R=\left[\begin{array}{ccccc}
F_{1} & { }_{1} M_{2} & \ldots & { }_{1} M_{n} & { }_{1} M_{*} \\
{ }_{2} M_{1} & F_{2} & \ldots & { }_{2} M_{n} & { }_{2} M_{*} \\
\vdots & \vdots & \ddots & \vdots & \vdots \\
{ }_{n} M_{1} & { }_{n} M_{2} & \ldots & F_{n} & { }_{n} M_{*} \\
0 & 0 & \ldots & 0 & F
\end{array}\right]=\begin{gathered}
P_{1} \\
\oplus \\
P_{2} \\
\oplus \\
\vdots \\
\oplus \\
P_{n} \\
\oplus \\
P_{*}
\end{gathered}
$$

such that $\operatorname{soc}\left(R_{R}\right)$ is an essential right ideal in $R$ isomorphic to a direct sum of finitely many copies of $P_{*}$ (called the right peak of $R$ ). Here $F_{1}, \ldots, F_{n}$ are local rings, $F=F_{*}$ is a division ring, ${ }_{i} M_{j}$ is an $F_{i}-F_{j}$-bimodule and the multiplication in $R$ is given by $F_{i}-F_{i}{ }^{-}$ bimodule maps $c_{i j i}:{ }_{i} M_{j} \otimes{ }_{j} M_{t} \rightarrow{ }_{i} M_{t}$ satisfying the natural associativity conditions. We denote by $P_{1}, \ldots, P_{n}, P_{*}$ the right indecomposable row ideals of $R$. Throughout we suppose that $R$ is basic, i.e. $R / J(R)$ is a product of division rings, where $J(R)$ is the Jacobson radical of $R$. We denote by $\bmod _{\mathrm{sp}}(R)$ the category of finitely generated socle projective right $R$-modules. The ring $R$ is called sp-representation-finite if the number of isomorphism classes of indecomposable modules in $\bmod _{\mathrm{sp}}(R)$ is finite.

We use the terminology and notation introduced in $[28,31]$.

Let us recall that if $I$ is a finite posed and $I^{*}=I \cup\{*\}$, where $i \prec *$ for all $i \in I$, then given a division ring $F$ the incidence algebra $F I^{*}$ is a right peak ring and $\bmod _{\mathrm{sp}}\left(F I^{*}\right)$ is 
equivalent to the category $I$-sp of $I$-spaces over $F[28,30]$. On the other hand, if $A$ is an $R$-order in a simple algebra $C$ over the field of fractions of $R$ then

$$
A_{C}=\left[\begin{array}{ll}
A & C \\
0 & C
\end{array}\right]
$$

is a noetherian right peak ring and the category latt $(A)$ of right $A$-lattices [26] is equivalent to the factor category $\bmod _{\mathrm{sp}}\left(A_{C}\right) /\left[P_{*}\right]=\operatorname{adj}\left(A_{C}\right)$ [31]. Let us also recall from [28] that if $\boldsymbol{K}_{\boldsymbol{F}}$ is a vector space category and $\boldsymbol{R}_{\boldsymbol{K}}$ is the right peak ring of $\boldsymbol{K}_{l}$ then there is a full dense functor $H: \mathscr{V}\left(\boldsymbol{K}_{F}\right) \rightarrow \bmod _{\mathrm{sp}}\left(\boldsymbol{R}_{\boldsymbol{K}}\right)$ preserving the representation type, where $\mathscr{V}\left(\boldsymbol{K}_{F}\right)$ is the factor space category of $\boldsymbol{K}_{F}$. This functor is frequently applied in the representation theory of algebras $[25,29]$.

One of the main aims of this paper is to show (Section 3) that under suitable assumptions on $R$ there is a preprojective component $\mathscr{P}_{\mathrm{sp}}(R)$ in the Auslander-Reiten valued quiver $\Gamma_{\mathrm{sp}}(R)$ of $\bmod _{\mathrm{sp}}(R)$ (see Sections 2 and 3, compare with [28; Remark 7]). In case $R$ is sp-representation-finite this will provide a simple algorithm presented in Section 4 for constructing all indecomposable modules in $\bmod _{\mathrm{sp}}(R)$ in a way described in $[14]$ for $\bmod (R)$.

In Section 5 we show that if $R$ admits a splitting poset decomposition (5.0) of its valued poset $\left(I_{R}^{*}, d\right)$ then $\Gamma_{\mathrm{sp}}(R)$ can be glued from $\Gamma_{\mathrm{sp}}(A)$ and $\Gamma_{\mathrm{sp}}(B)$ along a single linear section, where $A$ and $B$ are right peak rings derived from $R(5.3)$.

We say that $R$ is a $P I$-ring if $R$ satisfies a polynomial identity, which in the case of $R$ artinian means that the division rings $F_{1} / J\left(F_{1}\right), \ldots, F_{n} / J\left(F_{n}\right), F$ are finite-dimensional over their centers. In case $R$ is artinian an important invariant of $R$ is its value scheme $\left(I_{R}^{*}, d\right)[28,29]$, where $I_{R}^{*}=\{1, \ldots, n, *=n+1\}$ and there is an arrow

$$
i \stackrel{\left(d_{i j}, d^{\prime}\right.}{i j)} \rightarrow j, \quad i \neq j
$$

iff $d_{i j}=$ length $\left({ }_{i} M_{j}\right)_{F_{j}}, d_{i j}^{\prime}=$ length ${ }_{F_{i}}\left({ }_{i} M_{j}\right)$ are nonzero. If the bimodules ${ }_{1} M_{*}, \ldots,{ }_{n} M_{*}$ are simple and $R$ is schurian (i.e. $F_{1}, \ldots, F_{n}$ are division rings) then $\left(I_{R}^{*}, d\right)$ is a valued poset [28; Prop. 2.3], with respect to the relation $i \prec j \Leftrightarrow{ }_{i} M_{j} \neq 0$.

In $[20,21]$ sp-representation-finite schurian right peak $P I$-rings $R$ are characterized in terms of $\left(I_{R}^{*}, \boldsymbol{d}\right)$ and it is proved that every indecomposable module $X=\left(X_{i},{ }_{j} \varphi_{i}\right)$ in $\bmod _{\text {sp }}(R)$ over such a ring $R$ is uniquely determined by its dimension vector

$$
\operatorname{dim} X=\left(x_{1}, \ldots, x_{n}, x_{*}\right)
$$

where $X_{i}=X e_{i}, x_{i}=\operatorname{dim}\left(X_{i}\right)_{F_{i}}$ and $e_{1}, \ldots, e_{n}, e_{*}$ are the standard matrix idempotents. The algorithm presented in Section 3 based on the construction (3.1) of $\mathscr{P}_{\mathrm{sp}}(R)$ allows us to calculate $\operatorname{dim} X$ in terms of the matrices $\left(d_{i j}\right),\left(d_{i j}^{i}\right)$ for every indecomposable $X$ in $\bmod _{\mathrm{sp}}(R)$, where $R$ is as above.

2. Preliminaries and notation. Let us recall that $\bmod _{\mathrm{sp}}(R)$ is closed under direct sums, summands, kernels, extensions and has enough projectives because $P_{1}, \ldots, P_{n}, P_{*}$ are
in $\bmod _{\mathrm{sp}}(R)$.
If $e=e_{1}+\ldots+e_{n}, A=e R e, M=e R(1-e)$ then

$$
R \cong\left[\begin{array}{cc}
A & { }_{A} M_{F} \\
0 & F
\end{array}\right]
$$

and the ring [28]

$$
R^{V}=\left[\begin{array}{ll}
F & M^{*} \\
0 & A
\end{array}\right], \quad M^{*}=\operatorname{Hom}_{F}\left(M_{F}, F\right)
$$

is a left peak $P I$-ring. If $R$ is an artinian $P I$-ring then there is a reflection duality [28; Corollary 2.7$],[33 ; 2.6]$

$$
D^{\bullet}=D \nabla: \bmod _{\mathrm{sp}}(R) \rightarrow \bmod _{\mathrm{sp}}\left(R^{\bullet}\right)
$$

where $R^{\bullet}=\left(\tilde{R}^{\nabla}\right)^{\text {op }}$ and $\tilde{R}^{\nabla}$ is the ring Morita dual to $R^{\nabla}$.

It follows that $\bmod _{\mathrm{sp}}(R)$ has enough sp-injective modules [28; Cor. 2.7]. Let us recall from [28] that $Q$ in $\bmod _{\mathrm{sp}}(R)$ is sp-injective if $Q$ is injective with respect to monomorphisms $q: X^{\prime} \rightarrow X$ in $\bmod _{\mathrm{sp}}(R)$ such that Coker $q$ is also in $\bmod _{\mathrm{sp}}(R)$.

We shall need the following result.

LEMMA 2.1. Let $R$ be an artinian schurian upper triangular right peak PI-ring of the form (1.1) and let ${ }_{i} M_{j}^{t}=\operatorname{Hom}_{F_{t}}\left({ }_{i} M_{j}, F_{t}\right)$, where $t=i, j$. Then

(a) $T=R^{\bullet}$ is an artinian schurian right peak PI-ring and the valued posed $\left(I_{T}^{*}, \tilde{\boldsymbol{d}}\right)$ of $T$ is obtained from $(\boldsymbol{I}, \boldsymbol{d})$ by reversing direction of all arrows between elements in $I_{R}=I_{R}^{*}-\{*\}$.

(b) The injective envelope $E^{(j)}$ of top $\left(P_{j}\right)$ in $\bmod (R)$ has the form

$$
E^{(j)}=\left({ }_{1} M_{j}^{j}, \ldots,{ }_{j-1} M_{j}^{j}, F_{j}, 0, \ldots, 0 ;{ }_{k} \psi_{i}^{i}\right)
$$

where ${ }_{k} \psi_{i}{ }_{k} M_{j}^{j} \otimes_{k} M_{i} \rightarrow{ }_{i} M_{j}^{j}$ is such that its $F_{j}$-dual corresponds via the isomorphism $\operatorname{Hom}_{F_{j}}\left({ }_{k} M_{j}^{j},{ }_{k} M_{i}\right) \cong \operatorname{Hom}_{F_{k}}\left({ }_{k} M_{i},{ }_{k} M_{j}\right)$ to the map $\bar{c}_{k i j}$ adjoint to $c_{k i j}$. Moreover, $\underline{\operatorname{dim}} E^{(j)}=\left(d_{1 j}^{\prime}, d_{2 j}^{\prime}, \ldots, d_{j-1 j}^{\prime}, 1,0, \ldots, 0\right)$.

(c) The modules

$$
Q^{(0)}=E\left(P_{*}\right), \quad Q^{(j)}=\nabla^{-1} E^{(j)}, \quad j=1, \ldots, n,
$$

form a complete list of isoclasses of indecomposable sp-injective modules in $\bmod _{\mathrm{sp}}(R)$ and

$$
\operatorname{dim} Q^{(j)}=\left(d_{j *} d_{1 *}^{\prime}-d_{1 j}^{\prime}, \ldots, d_{j *} d_{n *}^{\prime}-d_{n j}^{\prime}, d_{j *}\right),
$$

where $d_{0 i}=d_{i *}^{\prime}, d_{0 i}^{\prime}=d_{i *}$ and $d_{i 0}=d_{i 0}^{\prime}=0$ for $i \geqslant 1$.

Prool. (a) follows from [28; Proposition 2.5] and Proposition 2.4 below. (b) and the first part of (c) follow immediately from [28; Propositions 2.5 and 2.6]. For (2.3) first compute the forms (2.2) by applying the functor $\nabla^{-1}$ to $E^{(j)}$ in the category of finitely generated top injective right $R^{\nabla}$-modules (denoted by $\bmod _{\mathrm{ti}}\left(R^{\nabla}\right)$ ) and then compute the corresponding dimensions keeping in mind the following result proved in [13].

Propositron 2.4. If $F_{i}$ and $F_{j}$ are division rings finite-dimensional over their centers and ${ }_{i} N_{j}$ is a finite-dimensional $F_{i}-F_{j}$-bimodule then

$$
\operatorname{dim}_{F_{j}}\left(N_{i} N_{j}^{i}\right)=\operatorname{dim}\left({ }_{i} N_{j}\right)_{F_{j}} \text { and } \operatorname{dim}\left({ }_{i} N_{j}^{j}\right)_{F_{i}}=\operatorname{dim}_{F_{i}}\left({ }_{i} N_{j}\right) \text {. }
$$


In the study of $\bmod _{\mathrm{sp}}(R)$ it is very convenient to use almost split sequences and irreducible maps.

We recall from $[3,4]$ that a homomorphism $f: X \rightarrow Y$ in $\bmod _{\mathrm{sp}}(R)$ is irreducible if $f$ is neither a splittable monomorphism nor a splittable epimorphism and in any factorization $f=h g$ in $\bmod _{\mathrm{sp}}(R)$ either $g$ is a splittable monomorphism or $h$ is a splittable epimorphism. It is easy to check that for $X, Y$ indecomposable over an artinian ring $R$ the map $f: X \rightarrow Y$ is irreducible if and only if $f \in J(X, Y)-J^{2}(X, Y)$, where $J(X, Y)$ consists of all $f \in \operatorname{Hom}_{R}(X, Y)$ such that $\operatorname{id}_{X}-q f$ is invertible for all $g \in \operatorname{Hom}_{R}(Y, X)$ and $J^{2}(X, Y)$ consists of $f^{\prime}=f^{\prime} f^{\prime \prime} \in J(X, Y)$ with $f^{\prime} \in J(Z, Y), f^{\prime \prime} \in J(X, Z)$ for some $Z$. We note that (see [23; 2.5])

$$
\operatorname{Irr}(X, Y)=J(X, Y) / J^{2}(X, Y)
$$

is an $F(Y)-F(X)$-bimodule where $F(Z)=$ End $(Z) / J($ End $(Z))$. Following Auslander [2] and Ringel [24] we define the Auslander-Reiten valued quiver $\left(\Gamma_{\mathrm{sp}}(R), d\right) \operatorname{of}_{\bmod _{\mathrm{sp}}}(R)$ as the set of isoclasses $[X]$ of indecomposable modules $X$ in $\bmod _{\mathrm{sp}}(R)$ connected by oriented valued arrows

$$
[X] \stackrel{\left(d_{\left.X Y, d_{X Y}\right)}^{\longrightarrow}\right.}{\longrightarrow}[Y]
$$

when $X \nsucceq Y$ and the dimensions

$$
d_{X Y}=\operatorname{dim} \operatorname{Irr}(X, Y)_{F(X)}, \quad d_{X Y}^{\prime}=\operatorname{dim}_{F(Y)} \operatorname{Irr}(X, Y)
$$

are nonzero (see also $[18,19,23]$ ). This means that there are irreducible maps

$$
X \rightarrow Y^{d_{X X}}, \quad X^{d_{X Y}^{\prime}} \rightarrow Y
$$

in $\bmod _{\mathrm{sp}}(R)$. We note that $\left(\Gamma_{\mathrm{sp}}(R), d\right)$ has a unique maximal element $\left[E\left(P_{*}\right)\right]$ and a unique minimal element $\left[P_{*}\right]$

We recall from $[3,4]$ that an exact sequence $0 \rightarrow X \stackrel{f}{\rightarrow} Y \stackrel{g}{\rightarrow} Z \rightarrow 0$ in $\bmod _{\mathrm{sp}}(R)$ is said to be almost split if it does not split, $X, Y$ are indecomposable and in addition it has the following equivalent properties:

(a) $f$ is left almost split in the sense that given any map $h: X \rightarrow V$ in $\bmod _{\mathrm{sp}}(R)$ which is not a splittable monomorphism, there is a map $t: Y \rightarrow V$ such that $t f=h$.

(b) $g$ is right almost split in the sense that given $h: U \rightarrow Z$ in $\bmod _{\mathrm{sp}}(R)$ which is not a splittable epimorphism, there is a map $t: U \rightarrow Y$ such that $g t=h$.

Note that left almost split maps as well as right almost split maps are irreducible.

The reader is referred to $[4,25]$ for elementary facts about almost split sequences and irreducible maps in $\bmod _{\mathrm{sp}}(R)$

Let us recall that $P_{i} J(R) \subseteq P_{i}$ is irreducible and right almost split. Moreover, if $R$ is an artinian $P I$-ring and $\left.Q^{(j)}=\nabla^{-1} E^{*}\right)$ is the indecomposable sp-injective module (2.2) then the induced map

$$
\lambda_{j}: Q^{(j)} \rightarrow \nabla^{-1}\left(E^{(j)} / \operatorname{soc}\left(E^{(j)}\right)\right)
$$

is left almost split in $\bmod _{\text {sp }}(R)$ because $E^{(j)} \rightarrow E^{(j)} / \operatorname{soc}\left(E^{(j)}\right)$ is left almost split in $\bmod _{t i}\left(R^{\nabla}\right)$. Note also that if $P_{*} \rightarrow X$ is irreducible in $\bmod _{\mathrm{sp}}(R)$ and $X$ is indecomposable then $X$ is projective (see [27; Lemma 1.3]).

We say that $R$ has almost split sp-sequences if every indecomposable non-sp-injective socle projective module $X$ admits a right almost split sequence

$$
0 \rightarrow X \rightarrow Y \rightarrow \Delta^{-} X \rightarrow 0
$$

in $\bmod _{\mathrm{sp}}(R)$ and any non-projective indecomposable socle projective module $Z$ admits a left almost split sequence

$$
0 \rightarrow \Delta X \rightarrow Y \rightarrow Z \rightarrow 0
$$

in $\bmod _{\mathrm{sp}}(R)$. The terms are determined uniquely up to isomorphism.

We know from $[4,5,26]$ that any right peak artin algebra $R$ has almost split sp-sequences (see also [28; Corollary 3.7]). Although this is not true for arbitrary artinian $P I$-rings (see $[23 ; 2.5]$ ) it is true for sp-representation-finite right peak ones ([21; Prop. 6.3]) It is also known that if $R \simeq F I^{*}$ or $R \simeq A_{C}$ (defined in the Introduction) then $R$ has almost split sp-sequences $[25,26,30]$.

3. A preprojective component in the valued translation quiver $\Gamma_{\mathrm{sp}}(R)$. Throughout this section we suppose that $R$ is an artinian right peak ring having almost split sp-sequences. In particular we can take for $R$ a right peak artin algebra, or an incidence ring $F I^{*}$ of a finite poset $I^{*}$, or an sp-representation-finite right peak $P I$-ring.

Following Auslander [2] a full valued subquiver $\mathscr{C}$ of $\left(\Gamma_{\mathrm{sp}}(R), d\right)$ is called a connected component if $\mathscr{C}$ is closed under taking neighbours and for any $[X],[Y]$ in $\mathscr{C}$ there is a sequence $[X]=\left[X_{0}\right],\left[X_{1}\right], \ldots,\left[X_{p}\right]=[Y]$ in $\mathscr{C}$ where $X_{i}$ and $X_{i+1}$ are connected by an irreducible map for $i=0,1, \ldots, p-1$. Following $[4,17]$ we call a component $\mathscr{C}$ in $\left(\Gamma_{\mathrm{sp}}(R), d\right)$ preprojective (resp. preinjective) if $\mathscr{C}$ has no oriented cycles and any $[X]$ in $\mathscr{C}$ has the form $\left[\Delta^{-t} P\right]$ (resp. [ $\left.\Delta^{t} Q\right]$ ) for some $t \geqslant 0$ and some indecomposable projective module $P$ (resp. sp-injective module $Q$ ). The module $N$ in $\bmod _{\mathrm{sp}}(R)$ is said to be hereditary projective (resp. hereditary sp-injective) if every submodule of $N$ is projective (resp. every socle projective indecomposable module $Y$ such that $\operatorname{Hom}_{R}(N, Y) \neq 0$ is sp-injective).

Given a right peak ring $R$ as above we put (cf. $[6,7,8]$ )

$$
\mathscr{P}_{\mathrm{sp}}(R)=\bigcup_{j=0}^{\infty} \mathscr{P}_{j}^{\mathrm{sp}}, \quad \mathscr{Z}_{\mathrm{sp}}(R)=\bigcup_{j=0}^{\infty} \mathscr{2}_{j}^{\mathrm{sp}},
$$

where $\mathscr{P}_{0}^{\mathrm{sp}}=\left\{[P] \in \Gamma_{\mathrm{sp}}(R) \mid P\right.$ is hereditary projective $\}$ and if $\mathscr{P P}_{j}^{\mathrm{sp}}$ is defined we put $\mathscr{P}_{j+1}^{s p}=\Delta^{-} \mathscr{P}_{j}^{s p} \cup \mathscr{P}_{j+1}$, where $[P] \in \overline{\mathscr{P}}_{j+1}$ iff there is a chain of irreducible maps

$$
P_{0}^{\prime} \rightarrow P_{1}^{\prime} \rightarrow \ldots \rightarrow P_{t}^{\prime} \rightarrow P_{t+1}^{\prime}=P
$$

with $\left[P_{0}^{\prime}\right] \in \Delta^{-} \mathscr{P}_{j}^{\text {sp }}$ and $P_{1}^{\prime}, \ldots, P_{t+1}^{\prime}$ are indecomposable projective. We note that $\left[P_{*}\right] \in \mathscr{P}_{0}^{\text {sp }}$ and that $\mathscr{P P}_{j}^{\mathrm{sp}}$ is finite for all $j$. The sets $\mathscr{2}_{j}^{\mathrm{sp}}, j \geqslant 0$, are defined dually starting from $E\left(P_{*}\right)$ and hereditary sp-injective modules using $\Delta$

Throughout for the sake of simplicity we shall identify $X$ with its isomorphism 
class $[X]$ and we shall write $J\left(P_{i}\right)$ instead of $P_{i} J(R)$. We shall write $\Gamma_{\mathrm{sp}}(R)$ instead of $\left(\Gamma_{\mathrm{sp}}(R), d\right)$.

We know from $[24 ; 3.4]$ (see also $[12,18,19]$ ) that there are natural ring isomorphisms $\tau_{Y}: F(Y) \rightarrow F(\Delta Y)$, where $F(Z)=$ End $(Z) / J$ End $(Z)$, and a nondegenerate bilinear form

$$
\chi_{X Y}: \operatorname{Irr}(\Delta Y, X) \otimes_{F(Y)} \operatorname{Irr}(X, Y) \rightarrow F(X)
$$

which induces an $F(Y)-F(X)$-bimodule isomorphism

$$
\operatorname{Irr}(X, Y) \cong \operatorname{Hom}_{F(X)}(\operatorname{Irr}(\triangle Y, X), F(X))
$$

for any indecomposable modules $X, Y$ in $\bmod _{\mathrm{sp}}(R), \Delta Y \neq 0$. Moreover, if $\Delta X \neq 0$ then $\Delta$ induces an $F(Y)-F(X)$-bimodule isomorphism

$$
\operatorname{Irr}(X, Y) \cong \operatorname{Irr}(\Delta X, \Delta Y)
$$

over ring isomorphisms $\tau_{Y}$ and $\tau_{X}$

We shall show below that $\left(\Gamma_{\mathrm{sp}}(R), \boldsymbol{d}, \Delta, \chi\right)$ is a valued translation quiver in the sense

of $[15,24,18]$; we call it the valued translation quiver of $\bmod _{\mathrm{sp}}(R)$.

We notice that if we suppose that $R$ is a $P I$-ring then $F(X)$ and $F(Y)$ are division $P I$-rings and in view of Proposition 2.4 the isomorphism (3.4) yields

$$
\left(d_{X Y}^{\prime}, d_{X Y}\right)=\left(d_{\Delta Y X}, d_{\Delta Y X}^{\prime}\right)
$$

Now we are able to prove one of the main results of this paper. It generalizes some of the results in $[9,11]$.

THEOREM 3.7. Let $R$ be a schurian artinian right peak riny having almost split sp-sequences. Moreover, suppose that for every $j$ there are an indecomposable module $T$ and $g_{j} \geqslant 0$ such that $J\left(P_{j}\right) \cong T_{j}^{g_{j}}$. Then $\mathscr{P}_{\mathrm{sp}}=\mathscr{P}_{\mathrm{sp}}(R)$ is a preprojective component of $\Gamma_{\mathrm{sp}}(R)$. If, in addition, $R^{\nabla}$ has a Morita duality then the functor $D^{\bullet}: \bmod _{\mathrm{sp}}(R) \rightarrow\left(\bmod _{\mathrm{sp}}\left(R^{\bullet}\right)\right)^{\mathrm{op}}(2.0)$ carries $\mathscr{P}_{\mathrm{sp}}(R)$ to the preinjective component $\mathscr{Q}_{\text {sp }}\left(R^{\bullet}\right)$.

Proof. We follow an idea of Bautista-Larrion [7]. We proceed in several steps. $1^{\circ} \mathscr{P}_{u}^{\text {su }} \cap \mathscr{P P}_{v}^{\text {sp }}$ is empty for $u<v$. We apply induction on $u$. Let $u=0$ and let $X \in \mathscr{P P}_{0}^{\text {sp }} \cap \mathscr{P P}_{v}^{\text {sp }}$. It follows that $X \notin \Delta^{-} \mathscr{P}_{v-1}^{\text {sp }}$ and therefore $X \in \mathscr{P}_{v}$. Then there exists a sequence (3.2) with $P_{0}^{\prime} \in \Delta^{-} \mathscr{P P}_{v-1}^{\mathrm{sp}}$. Since $X$ is hereditary projective, $P_{t}^{\prime}, \ldots, P_{0}^{\prime}$ are projective and therefore $P_{0}^{\prime} \notin \Delta^{-} \mathscr{P P}_{v-1}^{\mathrm{sp}}$; a contradiction.

Suppose that $1^{\circ}$ is proved for $u^{\prime}<u$ and let $X \in \mathscr{P}_{u}^{\text {sp }} \cap \mathscr{P}_{n}^{\text {sp }}$. If $X$ is not projective then $\triangle X \in \mathscr{P}_{u-1}^{\text {sp }} \cap \mathscr{P}_{v-1}^{\text {sp }}$ and we get a contradiction. If $X$ is projective then $X \in \mathscr{P P}_{n} \cap \mathscr{P}_{v}$ and there are two chains of irreducible maps

$$
P_{0}^{\prime} \rightarrow P_{1}^{\prime} \rightarrow \ldots \rightarrow P_{t}^{\prime} \rightarrow P_{t+1}^{\prime}=X, \quad P_{0}^{\prime \prime} \rightarrow P_{1}^{\prime \prime} \rightarrow \ldots \rightarrow P_{l}^{\prime \prime} \rightarrow P_{l+1}^{\prime \prime}=X,
$$

where $P_{j}^{\prime}, P_{j}^{\prime \prime} \in \overline{\mathscr{P}}_{u} \cap \overline{\mathscr{P}}_{v}$ are indecomposable projective for $j>0$ and $P_{0}^{\prime} \in \Delta^{--} \mathscr{P P P}_{u-1}^{\mathrm{sp}}$ $P_{0}^{\prime \prime} \in \Delta^{-} \mathscr{P}_{v-1}^{\mathrm{sp}}$. By our assumption, $J\left(P_{j}^{\prime}\right) \cong T_{j}^{\prime \theta_{j}^{\prime}}$ and $J\left(P_{j}^{\prime \prime}\right) \cong T_{j}^{g_{j}}$ for $j>0$, where $T_{j}^{\prime}$ and $T_{j}$ are indecomposable. Since $P_{i}^{\prime} \rightarrow P_{i+1}^{\prime}$ and $P_{i}^{\prime \prime} \rightarrow P_{i+1}^{\prime \prime}$ are irreducible, $P_{i}^{\prime} \cong T_{i+1}^{\prime}$ $P_{i}^{\prime \prime} \cong T_{i+1}$ for all $i$ and $T_{t+1}^{\prime} \cong T_{l+1}$. Hence, if $t \geqslant l$ then $P_{t}^{\prime} \cong P_{l}^{\prime \prime}, \ldots, P_{t-l}^{\prime} \cong P_{0}^{\prime \prime}$ and therefore $t=l$ because otherwise $\Delta P_{0}^{\prime \prime}=0$ and $P_{0}^{\prime} \notin \Delta^{-} \mathscr{P}_{v-1}^{\text {sp }}$, a contradiction. Consequently, $t=l, P_{0}^{\prime} \cong P_{0}^{\prime \prime} \in \Delta^{-}\left(\mathscr{P}_{u-1}^{\text {sp }} \cap \mathscr{P}_{v-1}^{\text {sp }}\right)$ and therefore $\mathscr{P}_{u-1}^{\text {sp }} \cap \mathscr{P}_{v-1}^{\text {sp }}$ is not empty contrary to the inductive assumption. This finishes the proof of $1^{\circ}$.

$2^{\circ}$ For any $j \geqslant 0, \mathscr{O P S p}_{j}$ is a section in the sense of Bautista $[6,7,8]$. We will prove that

(i) If $X \in \mathscr{P P}_{j}^{\prime \prime}$ then $\Delta X \notin \mathscr{P}_{j}^{\mathrm{sp}}$

(ii) If $X \rightarrow Y$ is an irreducible map, $X, Y$ are indecomposable in $\bmod _{\mathrm{sp}}(R)$ and $X \in \mathscr{P}_{j}^{\mathrm{sp}}$ then either $Y \in \mathscr{P}_{j}^{\mathrm{sp}}$ or $Y$ is not projective and $\triangle Y \in \mathscr{P}_{j}^{\mathrm{sp}}$.

The claim is obvious for $j=0$. Suppose it is proved for $i \leqslant j$. The claim is obvious for $j=0$. Suppose it is proved for $i \leqslant j$. The statement (i) for $j+1$ follows immediately from $1^{\circ}$. In order to prove (ii) for $j+1$ we suppose that $X \rightarrow Y$ is irreducible in $\bmod _{\mathrm{sp}}(R)$ and $X \in \mathscr{P} \mathbb{P}_{j+1}$.

First suppose $X$ is projective. If $Y$ is projective we are done. Otherwise $\Delta Y \neq 0$ and by (3.4) there is an irreducible map $\Delta Y \rightarrow X$. By our assumption, $J(X) \cong T^{g}$ for some indecomposable module $T$. It then follows that $\Delta Y \cong T$. On the other hand, since $X$ is projective, $X \in \overline{\mathscr{P}}_{j+1}$ and therefore there is a sequence (3.2) with $X=P$. Since $P_{t}^{\prime} \rightarrow X$ is irreducible, $P_{t}^{\prime} \cong T \cong \Delta Y$. Since $P_{t}^{\prime} \in \mathscr{P}_{j}^{\text {sp }}$, we have $Y=\Delta^{-} P_{t}^{\prime} \in \mathscr{P}_{j+1}^{\text {sp }}$ and (ii) follows.

Next suppose that $X$ is not projective. If $Y$ is projective then $Y \in \mathscr{P}_{j+1}$ and we are done. Otherwise, according to (3.5) there is an irreducible map $\Delta X \rightarrow \Delta Y$ with $\Delta X \in \mathscr{P}_{j}^{\text {sp }}$ and (ii) follows from the inductive assumption. This finishes the proof of $2^{\circ}$.

$3^{\circ}$ gpsp has no oriented cycles for $j \geqslant 0$. This is obvious for $j=0$ because otherwise there is a proper monomorphism $P_{i} \rightarrow P_{i}$ for some $i \in I_{R}$; a contradiction. Let $j>0$ and suppose, on the contrary, that $\mathscr{P P}_{j}^{\text {sp }}$ has an oriented cycle of irreducible maps

$$
X_{0} \rightarrow X_{1} \rightarrow \ldots \rightarrow X_{k}=X_{0}
$$

where $X_{1}, \ldots, X_{k}$ are indecomposable. Assume that $j$ is minimal with respect to this property. It follows that some $X_{i}$ is projective because otherwise $\Delta(*)$ is an oriented cycle of irreducible maps in $\mathscr{P P s p}_{j-1}$, contrary to our choice of $j$. If $X_{i}$ is projective then by our assumption, $J\left(X_{i}\right) \cong T^{y}$ with $T$ indecomposable and therefore $T \cong X_{i-1(\operatorname{modulo} k)}$ because there is an irreducible map $X_{i-1} \rightarrow X_{i}$. It follows from $2^{\circ}$ (ii) that $X_{i+1 \text { (modulok) }}$ is projective because otherwise according to (3.4) there is an irreducible map $\Delta X_{i+1} \rightarrow X_{i}$ and therefore $\Delta X_{i+1} \cong T \cong X_{i-1} \in \mathscr{P P}_{j}^{\mathrm{sp}} ;$ a contradiction with $2^{\circ}$ (i). This proves that $(*)$ consists of proper monomorphisms between indecomposable projective modules. This contradiction finishes the proof of $3^{\circ}$.

$4^{\circ}$ If $X \rightarrow Y$ is an irreducible map in $\bmod _{\mathrm{sp}}(R)$ and $X, Y$ are indecomposable with $Y \in \mathcal{P}_{j}$, then either $X \in \mathcal{P S O}_{j}$. For, if $Y$ is projective then $Y \in \mathbb{P}_{j}$ and therefore there is a chain (3.2) with $P_{0}^{\prime} \in \Delta^{-} P_{j}^{p p p}$. Since $J(Y) \cong T^{\theta}$ with $T$ indecomposable, $X \cong T \cong P_{l}^{\prime} \in \mathscr{P}_{j}^{\mathrm{sp}}$ as required. If $Y$ is not projective then $Y \in \Delta^{-} \mathscr{P P}_{j-1}^{\mathrm{sp}}$ and according to (3.4) there is an irreducible map $\Delta Y \rightarrow X$. Since $\Delta Y \in \mathscr{P}_{j-1}^{\text {sp }}$, by $2^{\circ}$ (ii) either $X \in \mathscr{P}_{j-1}^{\text {sp }}$ or $X$ is not projective and $X \in \Delta^{-} \mathscr{P P P}_{j-1}^{\text {sp }} \subseteq \mathscr{P}_{j}^{\text {sp. }}$. Thus $4^{\circ}$ is proved.

$5^{\circ} \mathscr{P}_{\mathrm{sp}}$ is a preprojective component. It is easy to conclude from $1^{\circ}, 3^{\circ}$ and $4^{\circ}$ that $\mathscr{P}_{\mathrm{sp}}$ has no oriented cycles. Further, if $Y \in \mathscr{P P}_{j}^{\text {sp }}$ is not projective then the existence of a left almost split sp-sequence ending with $Y$ yields the existence of irreducible maps $\Delta Y \rightarrow Y^{\prime} \rightarrow Y$ with $Y^{\prime}$ indecomposable and in view of $2^{\circ}$ and $4^{\circ}, \Delta Y \in \mathscr{P}_{i}^{\text {sp }}$ for some $i<j$. 
Continuing this way we will find $t \in N$ such that $\Delta^{t} Y$ is projective because otherwise we get a contradiction with $2^{\circ}$.

The remaining part of the proposition easily follows from the duality (2.0).

Note that Theorem 3.7 applies to PI-rings satisfying the conditions (i), (ii) below because of the following result.

PROPOSITION 3.8. Let $R$ be schurian basic artinian right peak PI-ring (1.1) such that

(i) $d_{i *} d_{j *}^{\prime} \leqslant 3$ for $j=1, \ldots, n$;

(ii) the valued poset $\left(I_{R}^{*}, d\right)$ does not contain as an upper valued subposet one of the posets

$$
\widetilde{G}_{2}^{\prime}: 0 \rightarrow \circ \stackrel{(1,3)}{\rightarrow} *, \quad \bar{G}_{2}^{\prime \prime}: \circ \rightarrow 0^{(3,1)} \rightarrow *
$$

Then $R$ is isomorphic to an upper triangular form (1.1) and for every $P_{i}=e_{i} R, i=1, \ldots, n$, there is an indecomposable module $T_{i}=T\left(P_{i}\right)$ such that $F\left(T_{i}\right)=\operatorname{End}\left(T_{i}\right)$ is a division ring,

$$
J\left(P_{i}\right)=T_{i}^{g_{i}}
$$

with $g_{i}=\operatorname{GCD}\left(d_{i i+1}, \ldots, d_{i *}\right)$, and

$\left(\operatorname{dim}_{F_{i}} \operatorname{Hom}_{R}\left(T_{i}, P_{i}\right), \operatorname{dim} \operatorname{Hom}_{R}\left(T_{i}, P_{i}\right)_{F\left(T_{i}\right)}\right)= \begin{cases}\left(d_{j *}^{\prime}, d_{j *}\right) & \text { if } d_{j k}=d_{j *} d_{k *}^{\prime} \text { for all } k \succ j, \\ (1,1) & \text { otherwise. }\end{cases}$

For every $Q^{(j)}, j=1, \ldots, n$, there exists an indecomposable module $\hat{T}_{j}$ such that $F\left(\hat{T}_{j}\right)$ is $a$ division ring and

where

$$
\nabla^{-1}\left(E^{(j)} / \operatorname{soc} E^{(j)}\right) \cong \hat{T}_{j}^{j^{j}}
$$

$$
g_{j}=\operatorname{GCD}\left(d_{j *} d_{1 *}^{\prime}-d_{1 j}^{\prime}, \ldots, d_{j *} d_{j-1 *}^{\prime}-d_{j-1 j}^{\prime}, d_{j *} d_{j *}^{\prime}-d_{j j}^{\prime}+1,\right.
$$

$$
\left.d_{j *} d_{j+1 *}^{\prime}-d_{j+1 j}^{\prime}, \ldots, d_{j *} d_{n *}^{\prime}-d_{n j}^{\prime}, d_{j *}\right) \quad \text { (see 2.6), }
$$

$\left(\operatorname{dim}_{F\left(\hat{T}_{j}\right)} \operatorname{Hom}_{R}\left(Q^{(j)}, \hat{T}_{j}\right), \operatorname{dim} \operatorname{Hom}_{R}\left(Q^{(j)}, \hat{T}_{j}\right)_{F\left(Q^{(j)}\right)}\right)= \begin{cases}\left(d_{j *}, d_{j *}^{\prime}\right) & \text { if } d_{i j}=d_{i *} d_{j *}^{\prime} \text { for all } i \prec j, \\ (1,1) & \text { otherwise. }\end{cases}$

Proof. The first statement follows from [21, Proposition 7.1] and its proof. The second follows from the first in view of Lemma 2.1 (a) and the duality (2.0) which carries the map (2.6) to the map $J\left(P_{j}\right) \subset P_{j}$ in $\bmod _{\mathrm{sp}}\left(R^{\circ}\right)$.

By Theorem 3.7 and Proposition 3.8 we get

Corollary 3.9. If $R$ is a schurian artinian right peak PI-ring (1.1) satisfying conditions (i), (ii) above and having almost split sp-sequences then $\mathscr{P}_{\mathrm{sp}}(R)$ is a preprojective component of $\Gamma_{\mathrm{sp}}(R)$. In particular, this happens if $R$ is an $\mathrm{sp}$-representation-finite schurian PI-ring $[21 ; 2.10]$.

From Theorem 3.7 and its proof immediately follows

Corollary 3.10. Let $R$ be as in Theorem 3.7. Then (a) $X \in \mathscr{P}_{\mathrm{sp}}(R)$ if and only if $X$ is sp-preprojective in the sense that the number of nonisomorphic indecomposable modules $Z$ in $\bmod _{\mathrm{sp}}(R)$ with $\operatorname{Hom}_{R}(Z, X) \neq 0$ is finite.

(b) $Y$ belongs to a preinjective component of $R$ if and only if $Y$ is sp-preinjective in the sense that the number of nonisomorphic indecomposable modules $Z$ in $\bmod _{\mathrm{sp}}(R)$ with $\operatorname{Hom}_{R}(Y, \mathrm{Z}) \neq 0$ is finite.

Applying well-known arguments of Auslander [2] we get

COROLLARY 3.11. Let $R$ be as in Theorem 3.7. Then the following conditions are equivalent:

(a) $R$ is sp-representation-finite.

(b) $\left(\Gamma_{\mathrm{sp}}(R), d\right)=\mathscr{P}_{\mathrm{sp}}(R)$.

(c) Every module in $\bmod _{\mathrm{sp}}(R)$ is sp-preinjective.

(d) $\mathscr{P}_{\mathrm{sp}}(R)$ is finite.

(e) $E\left(P_{*}\right)$ is sp-prepojective.

Remark 3.12. It follows from the corollaries above that if $R$ is sp-representation-infinite having the duality $(2.0)$ then $\Gamma_{\mathrm{sp}}(R)$ has the following shape (like in the hereditary case [25]):

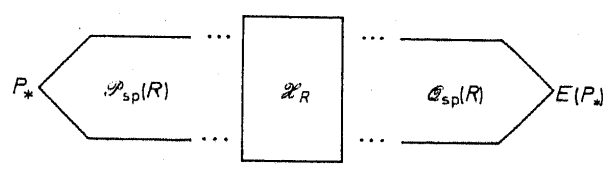

where $\mathscr{X}_{R}$ is a disjoint union of components and there are no maps from modules in $\mathscr{2}_{\mathrm{sp}}(R)$ (resp. in $\mathscr{X}_{R}$ ) to modules in $\mathscr{P}_{\mathrm{sp}}(R) \cup \mathscr{X}_{R}$ (resp. in $\mathscr{P}_{\mathrm{sp}}(R)$ ). In case $R$ is sp-representation-finite $\mathscr{X}_{R}$ is empty and $\mathscr{P}_{\mathrm{sp}}(R)=\mathscr{Q}_{\mathrm{sp}}(R)$.

Now we are able to prove an sp-counterpart of a result of Bautista-LarrionSalmeron [7, 8] (see also Ringel [25]).

Proposition 3.13. Let $R$ be an artinian schurian basic right peak PI-ring having almost split sp-sequences and such that $d_{j_{*}} d_{j *}^{\prime} \leqslant 3$ for all $j \in I_{R}$ and $\left(I_{R}^{*}, \boldsymbol{d}\right)$ does not contain upper subposets $\widetilde{G}_{2}^{\prime}$ and $\widetilde{\boldsymbol{G}}_{2}^{\prime \prime}$. Then $\mathscr{P}_{\mathrm{sp}}(R)$ is a preprojective component of $\Gamma_{\mathrm{sp}}(R)$ and has the following properties:

(a) $\operatorname{Ext}_{R}^{1}(X, X)=0$ and $\operatorname{End}(X)$ is a division ring for all $X$ in $\mathscr{P}_{\mathrm{sp}}(R)$.

(b) For any arrow

$$
X \stackrel{\left(d_{X Y, d X Y)}^{\prime}\right.}{\longrightarrow} Y
$$

in $\mathcal{P}_{\mathrm{sp}}(R)$ there exists an indecomposable projective module $P$ with $J(P) \cong T^{\sharp}, T$ indecomposable, such that $\left(d_{X Y}^{\prime}, d_{X Y}\right)$ or $\left(d_{X Y}, d_{X Y}^{\prime}\right)$ is equal to $\left(d_{T P}^{\prime}, d_{T P}\right)$ and $d_{T P} d_{T P}^{\prime} \leqslant 3$.

(c) $\left(\mathscr{P}_{\mathrm{sp}}(R), \boldsymbol{d}, \Delta, \chi\right)$ is a symmetrizable valued translation quiver (i.e. it has no valued loops $\varsigma \circ$ and no minimal arrows $\stackrel{(d, d)}{\longrightarrow} 0$ with $d \geqslant 2[10,24,18])$ with trivial fundamental group in the sense of Bongartz-Gabriel $[10,15]$ (see also $[24,19]$ ).

(d) $F(X)=$ End $(X)$ is a division ring for every $X$ in $\mathscr{P}_{\mathrm{sp}}(R)$ and $\operatorname{Hom}_{R}(X, Y)$ is finite-dimensional over $F(X)$ as well as over $F(Y)$ for every $X, Y$ in $\mathscr{P}_{\mathrm{sp}}(R)$. 
Proof. We know from Corollary 3.9 that $\mathscr{P}_{\mathrm{sp}}=\mathscr{P}_{\mathrm{sp}}(R)$ is a preprojective component in $\Gamma_{\mathrm{sp}}(R)$.

(a) If $f \in \operatorname{End}(X)$ is noninvertible then $f$ is a sum of compositions of irreducible maps in $\bmod _{\mathrm{sp}}(R)$ because the direct sum of all modules $Y$ in $\mathscr{P}_{\text {sp }}$ with $\operatorname{Hom}_{R}(Y, X) \neq 0$ is finite and therefore has semiprimary endomorphism ring. Hence there is a cycle in $\mathscr{P}_{\mathrm{sp}}$, a contradiction. If $\operatorname{Ext}_{R}^{1}(X, X) \neq 0$ then by arguments used above there is a cycle in $\mathscr{P}_{\mathrm{sp}}$ and we again get a contradiction.

(b) Since $X, Y$ are in $\mathscr{P}_{\text {ap }}$ there is $t \geqslant 0$ such that the modules $\Delta^{t} X, \Delta^{t} Y$ are both nonzero and one of them is indecomposable projective. First suppose that $\Delta^{t} Y=P$ is projective. Since we know from Proposition 3.8 that $J(P) \cong T^{\prime \prime}$, where $g \geqslant 0$ and $T$ is indecomposable, (b) follows from (3.5). Next suppose that $\Delta^{t} Y$ is not projective and $\Delta^{t} X=P$ is projective. Again, by Proposition $3.8, J(P) \cong T^{y}$ where $g \geqslant 0$ and $T$ is indecomposable. Since according to (3.4) and (3.5) there is an irreducible map $\Delta^{t+1} Y \rightarrow \Delta^{t} X$ we have $\Delta^{t+1} Y \cong T$. Now (b) follows from (3.4)-(3.6).

(c) The first assertion follows from (b) and Proposition 3.8. The second immediately follows from the definition of the fundamental group because $\mathscr{P}_{\mathrm{sp}}$ has no oriented cycles and $J\left(P_{i}\right) \cong T_{i}^{g_{i}}$ for some $g_{i} \geqslant 0$ and $T$ indecomposable.

(d) If $X \cong Y$ are in $\mathscr{P}_{\text {sp }}$ then by the arguments used in the proof of (a) any $f \in \operatorname{Hom}_{R}(X, Y)$ is a sum of compositions of irreducible maps. It follows that for any $t \geqslant 1$ there is an $F(Y)-F(X)$-bimodule epimorphism

$$
\xi_{t}: \oplus_{i} \operatorname{Irr}\left(X_{i n_{i t}}, Y\right) \otimes_{F\left(X_{i i_{i t}}\right)} \ldots \otimes_{F\left(X_{i 1}\right)} \operatorname{Irr}\left(X, X_{i 1}\right) \rightarrow J^{t}(X, Y) / J^{t+1}(X, Y)
$$

where the sum is finite and the $X_{i j}$ are in $\mathscr{P}_{\mathrm{sp}}$. Since by Corollary $3.10, J^{m}(X, Y)=0$ for some $m$, in view of $\xi_{t}$, (d) follows from (b) by an easy induction on $t$. This completes the proof.

COROLLARY 3.14. Let $R$ be a basic artinian sp-representation-finite right peak PI-ring. Then the following conditions are equivalent:

(a) $\left(\Gamma_{\mathrm{sp}}(R), \boldsymbol{d}, \Delta, \chi\right)$ is a symmetrizable valued translation quiver and $R$ is simply sp-connected in the sense that the fundamental group of $\left(\Gamma_{\mathrm{sp}}(R), \boldsymbol{d}, \Delta, \chi\right)$ is trivial.

(b) $\left(\Gamma_{\mathrm{sp}}(R), d\right)$ has no oriented cycle.

(c) End $(X)$ is a division ring for every indecomposable module $X$ in $\bmod _{\mathrm{sp}}(R)$.

\section{(d) $R$ is schurian.}

Proof. (a) $\Rightarrow$ (b) and (c) $\Rightarrow$ (d) are obvious.

(b) $\Rightarrow$ (d). Apply arguments in the proof of Proposition 3.13(a).

(a) $\Leftarrow$ (d) $\Rightarrow$ (c). We know from [21; Proposition 6.3] that $R$ has almost split sp-sequences. Then by Corollary $3.9, \mathscr{P}_{\mathrm{sp}}(R)$ is a preprojective component and $\left(\Gamma_{\mathrm{sp}}(R), d\right)=\mathscr{P}_{\mathrm{sp}}(R)$ according to Corollary 3.11 . Consequently (a) and (c) follow from Proposition 3.12 and the proof is complete.

4. An algorithm for describing $\mathscr{P}_{\mathrm{sp}}(R)$. Suppose that $R$ is a basic schurian artinian right peak $P I$-ring of the form (1.1) which is upper triangular and the valued poset $\left(I_{R}^{*}, d\right)$ has the properties (i) and (ii) of Proposition 3.8. Then according to Corollary 3.9,
$\mathscr{H}_{\mathrm{sp}}(R)$ is a preprojective component and applying arguments of Happel [16] similarly to [21; Section 8] one can show that every indecomposable $X$ in $\mathscr{P}_{\mathrm{sp}}(R)$ is uniquely determined by its dimension vector $\operatorname{dim} X(1.3)$ provided $R$ is a finite-dimensional algebra over a field.

Following the method initiated by Bautista, Brenner and Ringel (see [14]) we can describe $\mathscr{P}_{\mathrm{sp}}(R)$ in terms of $\underline{\operatorname{dim}} X, X \in \mathscr{P}_{\mathrm{sp}}(R)$ as follows:

$\left(\mathrm{a}_{1}\right)$ Given $R$ of the form (1.1) which is upper triangular we calculate the numbers $d_{i j}, d_{i j}^{\prime}, i, j=1, \ldots, n, *(1.2)$ and we write two sets of vectors in $Z^{n+1}$ :

$$
\mathscr{P}=\left\{p_{1}, \ldots, p_{n}, p_{n+1}=p_{*}\right\}, \quad \mathscr{2}=\left\{q_{0}, \ldots, q_{n}\right\}
$$

where

$$
\begin{aligned}
& p_{j}=\operatorname{dim} P_{j}=\left(0, \ldots, 1, d_{j j+1}, \ldots, d_{j *}\right), \\
& q_{j}=\operatorname{dim} Q^{(j)}=\left(d_{j *} d_{1 *}^{\prime}-d_{1 j}^{\prime}, \ldots, d_{j *} d_{n *}^{\prime}-d_{n j}^{\prime}, d_{j *}\right)
\end{aligned}
$$

with $d_{0 i}=d_{i *}^{\prime}, d_{i 0}^{\prime}=d_{i *}$ and $d_{i 0}=d_{i 0}^{\prime}=0$ for all $i \geqslant 1$ (see 2.3).

$\left(\mathrm{a}_{2}\right)$ Consider the set $\mathscr{T}=\left\{t_{1}, \ldots, t_{n}\right\}$, where

$$
t_{j}=\boldsymbol{t}\left(p_{j}\right):=g_{j}^{-1} p_{j} \quad \text { with } g_{j}=\operatorname{GCD}\left(d_{j j+1}, \ldots, d_{j *}\right) .
$$

Note that according to Proposition 3.8 we have $J\left(P_{j}\right) \cong T\left(P_{j}\right)^{g_{s}}, t_{j}=\underline{\operatorname{dim}} T\left(P_{j}\right)$ and the valued arrow from $T\left(P_{j}\right)$ to $P_{j}$ in $\left(\Gamma_{\mathrm{sp}}(R), d\right)$ has the form

$$
\begin{array}{ll}
T\left(P_{j}\right) \stackrel{\left(d_{j * *}^{\prime}, d_{j *}\right)}{\longrightarrow} P_{j} & \text { if } d_{i k}=d_{j *} d_{k *}^{\prime} \text { for all } k \succ j, \\
T\left(P_{j}\right) \stackrel{(1,1)}{\longrightarrow} P_{j} & \text { otherwise. }
\end{array}
$$

$\left(a_{3}\right)$ We define inductively sets $l_{0}, l_{1}, \ldots, l_{m}$ of vectors in $Z^{n+1}$ and valued arrows between vectors of $l_{i}$ and $l_{i+1}$ as follows:

(i) We mark vectors of $l_{j}$ as pairwise different points on the line $x_{1}=j$ in the plane $\boldsymbol{R}^{2}$.

(ii) Let $l_{0}=\left\{p_{*}\right\}$ and define $l_{1}$ as the set of vectors $p_{i} \in \mathscr{P}$ such that $t\left(p_{i}\right)=p_{*}$. For any such $i$ we connect $p_{*}$ with $p_{i} \in l_{1}$ by the valued arrow

$$
p_{*} \stackrel{\left(d_{i *}^{\prime}, d_{i *}\right)}{\longrightarrow} p_{i}
$$

(iii) Given $x \in I_{k \sim 1}-2$ we form a mesh

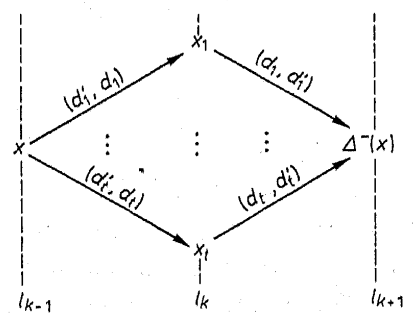


where $x_{1}, \ldots, x_{t}$ are all vectors in $l_{k}$ connected with $x, d_{j}^{\prime}=d_{x x_{j}}^{\prime}, d_{j}=d_{x x_{j}}$, $\Delta^{-}(x)=\sum_{j=1}^{t} x_{j} d_{j}^{\prime}-x$. We put

$$
l_{k+1}=\left\{\Delta^{-}(x) \mid x \in l_{k-1}-\mathscr{Q}\right\} \cup\left\{\boldsymbol{\sigma}_{j} \in \mathscr{P} ; \boldsymbol{t}\left(p_{j}\right) \in \mathscr{T} \cap l_{k}\right\} .
$$

We connect $x_{1}, \ldots, x_{t}$ with $\Delta^{-}(x)$ as is marked on the mesh above and we connect $t_{j}$ with $p_{j}$ by the valued arrow (4.2) with $T\left(P_{j}\right), P_{j}$ and $t_{j}, p_{j}$ interchanged.

Note that if $x=\operatorname{dim} X \in l_{k-1}-\mathscr{Q}$ and $X_{1}, \ldots, X_{t}$ are all indecomposable modules in $\bmod _{\mathrm{sp}}(R)$ connected by irreducible maps starting from $X$, then taking $x_{j}=\underline{\operatorname{dim}} X_{j}$ we get $\Delta^{-}(x)=\underline{\operatorname{dim}} \Delta^{-}(X)$.

This together with the remark in (ii) shows that $\mathscr{P}_{\mathrm{sp}}(R)=\bigcup_{j=0}^{\infty} l_{j}$ if we identify $X$ with $\underline{\operatorname{dim}} X$.

(4.3) Conclusion. The procedure will stop at step $m$ iff $q_{0}=\operatorname{dim} E\left(P_{*}\right) \in l_{m}$. In this case $R$ is sp-representation-finite and $\left(\Gamma_{\mathrm{sp}}(R), d\right)=l_{0} \cup \ldots \cup l_{m}$ (Corollary 3.11). Thus we get a simple procedure for describing $\mathscr{P}_{\text {sp }}(R)$ (and dually for describing the preinjective component $\mathscr{Q}_{\mathrm{sp}}(R)$ ) which is the Auslander-Reiten quiver provided $R$ is sp-representation-finite.

EXAMPLE 4.4. Let $\boldsymbol{R}$ denote the real numbers and $\boldsymbol{C}$ the complex numbers. Consider the $7 \times 7$ matrix ring

$$
T=\left[\begin{array}{llllllll}
R & R & C & R & C & R & C \\
& R & C & R & C & R & C \\
& C & 0 & C & 0 & C \\
& & & R & C & R & C \\
& & & & C & 0 & C \\
& & & & & & R & C \\
0 & & & & & & C
\end{array}\right]
$$

where the maps $c_{i j k}$ are given by the multiplication in $C$. Then $T$ is a right peak $\boldsymbol{R}$-algebra which is sp-representation-finite and

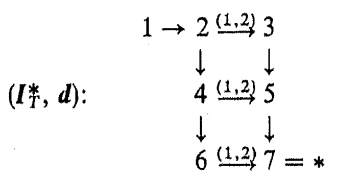

Applying the algorithm one can construct $\left(\Gamma_{\mathrm{sp}}(T), d\right)$ presented in Figure 1.

Other examples can be found in [21; Appendix] and in [30].

5. The Auslander-Reiten quiver $\Gamma_{\mathrm{sp}}(R)$ of a splitting ring. Suppose that $R$ is an artinian right peak $P I$-ring of the form (1.1) which is upper triangular and has almost split sp-sequences. In particular, $R$ has the reflection duality (2.0). Moreover, we suppose that $\left(I_{R}^{*}, d\right)$ is a valued posed with respect to the relation $i \prec j \Leftrightarrow{ }_{i} M_{j} \neq 0$. Following [21;

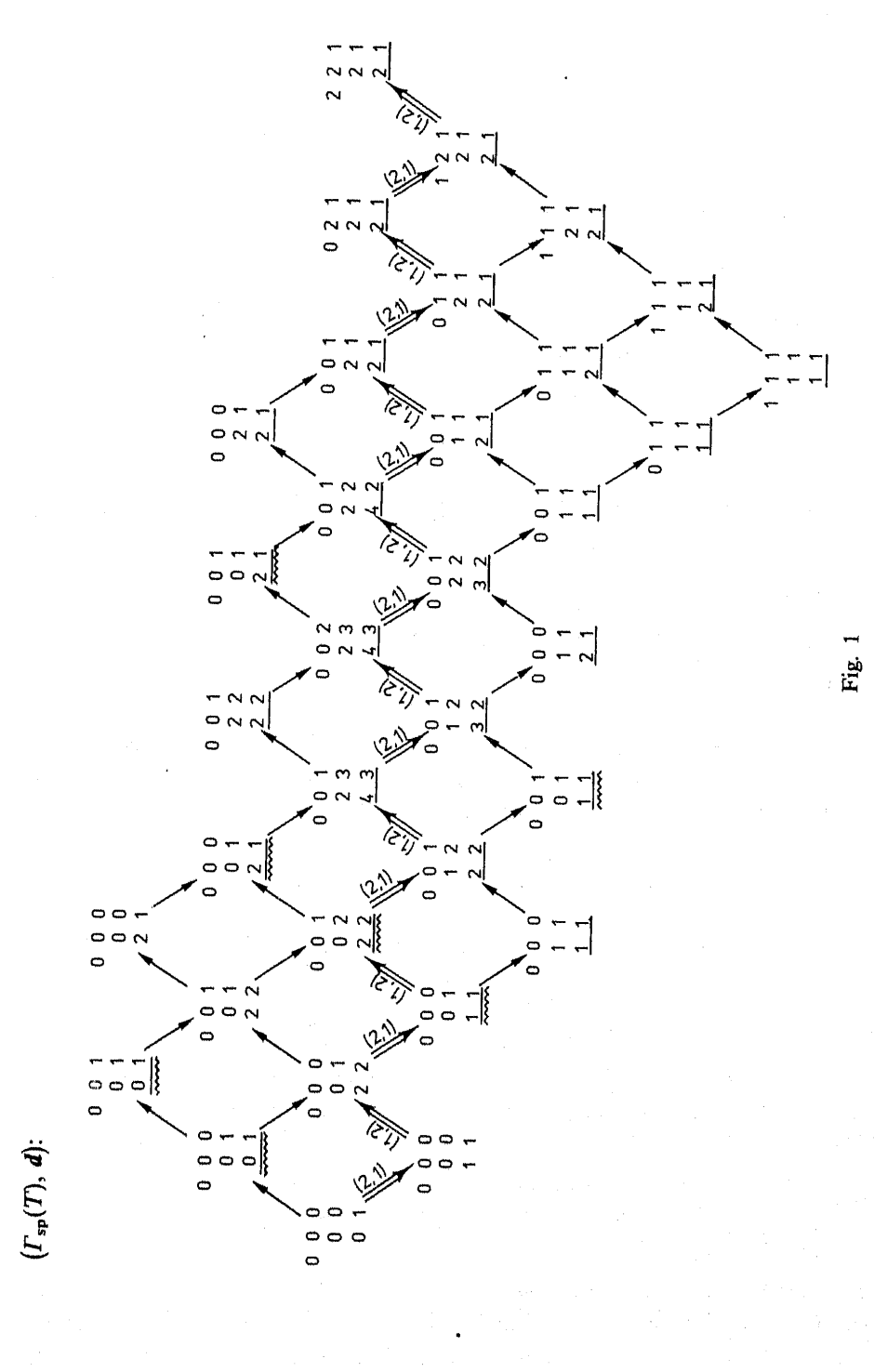


Definition 4.5] we say that $\left(I_{R}^{*}, d\right)$ has a splitting decomposition if there is a disjoint union poset decomposition

$$
I_{R}=I^{\prime}+C+I^{\prime \prime}
$$

such that $I^{\prime}, I^{\prime \prime}$ are not empty and

(i) $i^{\prime} \prec i^{\prime \prime}$ for all $i^{\prime} \in I^{\prime}, i^{\prime \prime} \in I^{\prime \prime}$ and there are no relations $c \prec i^{\prime}, i^{\prime \prime} \prec c$ with $c \in C$, $i^{\prime} \in I^{\prime}, i^{\prime \prime} \in I^{\prime \prime}$,

(ii) $\left(C^{*}, d\right)$ with $C^{*}=C \cup\{*\}$ is a homogeneous chain

$$
\left(C^{*}, d\right): c_{1} \rightarrow c_{2} \rightarrow \ldots \rightarrow c_{m} \rightarrow c_{m+1}=*, \quad d_{c_{j} c_{j+1}}=d_{c_{j} c_{j+1}}^{\prime}=1,
$$

(iii) $d_{i j}=d_{i *} d_{j *}^{\prime}$ for all $i \in I^{\prime}, j \in I^{\prime \prime}$.

In this case $\left(I_{R}^{*}, d\right)$ has the form

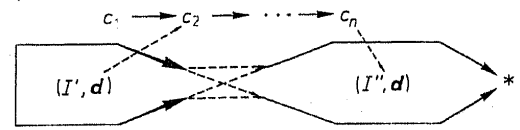

Throughout suppose that $\left(I_{R}^{*}, d\right)$ admits a splitting poset decomposition (5.0) and put

(5.2) $\quad e=e_{*}+e\left(I^{\prime}+C\right), \quad \eta=e_{*}+e\left(C+I^{\prime \prime}\right), \quad e^{\prime}=e\left(I^{\prime}\right), \quad e^{\prime \prime}=e_{*}+e\left(I^{\prime \prime}\right)$,

$J^{*}=J \cup\{*\}$ where $e(J)=\sum_{j \in J} e_{j}$ for $J \subseteq I_{R}$. Moreover, we put

$$
A=e R e, \quad B=\eta R \eta \text {. }
$$

Note that $A$ and $B$ are the right peak ring $R_{I^{\prime}+C}$ and $R_{C+I^{\prime \prime}}[29 ; 1.13]$ obtained from the form (1.1) of $R$ by omitting all rows and columns with indices in $I^{\prime \prime}$ and $I^{\prime}$, respectively. Following $[28,29,21]$ consider the functors (see [1])

$$
\bmod _{\mathrm{sp}}(A) \underset{r_{A}}{\stackrel{L}{\rightleftarrows}} \bmod _{\mathrm{sp}}(R) \underset{r_{B}}{\stackrel{T}{\leftrightarrows}} \bmod _{\mathrm{sp}}(B),
$$

where $\boldsymbol{r}_{A}=\boldsymbol{r}_{I^{\prime}+C}, \boldsymbol{r}_{B}=\boldsymbol{r}_{C+I^{\prime \prime}}$ are the restriction functors to $\left(I^{\prime}+C\right)^{*},\left(C+I^{\prime \prime}\right)^{*}$, i.e. $r_{A}(X)=X e=\left(X_{i},{ }_{j} \varphi_{i}\right)_{i, j \in\left(I^{\prime}+C\right)^{*}}$,

$$
L\left(Y_{A}\right)=\operatorname{Hom}_{A}\left(R e, Y_{A}\right), \quad T\left(Z_{B}\right)=Z \otimes_{B} \eta R .
$$

Following [32; 5.15] and [33] we are going to show that $\left(I_{\mathrm{sp}}(R), d\right)$ is a simple glueing of $\left(\Gamma_{\mathrm{sp}}(A), d\right)$ and $\left(\Gamma_{\mathrm{sp}}(B), d\right)$ by applying the functors $L$ and $T$.

The basic role is played by the following result.

Proposition 5.6. Under the assumptions and notations above we have:

(a) The functors $T, L$ are full, faithful and $r_{A} L \cong \mathrm{id}, r_{B} T \cong$ id. $T$ is left adjoint to $r_{B}$ and $L$ is right adjoint to $r_{A}$.

(b) If $Y_{A}$ is indecomposable in $\bmod _{\mathrm{sp}}(A)$ then the restriction $(L Y) e^{\prime \prime}$ of $L Y$ to $I^{\prime \prime} \cup\{*\}$ is isomorphic to $E^{\prime \prime}\left(\operatorname{soc} Y e^{\prime \prime}\right) \cong E^{\prime \prime}\left(P_{*}\right)^{h}$, where $h=\operatorname{dim}\left(Y e_{*}\right)_{F}$ and $E^{\prime \prime}\left(P_{*}\right)=E_{B}\left(P_{*}\right) e^{\prime \prime}$ is the injective envelope of $P_{*}$ in $\bmod _{\mathrm{sp}}\left(e^{\prime \prime} R e^{\prime \prime}\right)$. If $Y e^{\prime}=0$ then $Y \cong P_{c_{j}}$ for some $j=1, \ldots, m+1$, where $c_{m+1}=*$. (c) $T$ is exact, $T\left(e_{i} B\right)=e_{i} R$ for $i \in\left(C+I^{\prime \prime}\right)^{*}$ and $T\left(Q_{(j)}^{(j)} \cong Q^{(j)}\right.$ for $j \in I^{\prime \prime}$.

(d) $L$ is exact, $L\left(e_{i} A\right)=e_{i} R$ for $i \in I^{\prime}$ and $\left.L Q_{A}^{(j)}\right) \cong Q^{(j)}$ for $j \in I^{\prime}+C$.

(e) If $X$ is indecomposable in $\bmod _{\mathrm{sp}}(R)$ and $X e^{\prime} \neq 0$ then $r_{A}(X)$ is indecomposable and $\boldsymbol{L r}_{A}(Y) \cong X$. If $X e^{\prime}=0$ then $\boldsymbol{r}_{B}(X)$ is indecomposable and $\operatorname{Tr}_{B}(X) \cong X$. Every indecomposable module $X$ in $\bmod _{\mathrm{sp}}(R)$ is in one of the images $\operatorname{Im} T, \operatorname{Im} L$.

Proof. (a) is well known (see [1]).

(b) Since $L$ is right adjoint to $r_{A}$ it carries injectives to injectives. Then the first part of (b) follows by the arguments in the proof of [28; Prop. 2.5 (a)]. The second part of (b) and the first two statements in (c) follow immediately from the definitions.

(e) It follows from [21; Lemma 4.6] that the splitting poset decomposition (5.0) induces a corresponding ring splitting decomposition of $R$ [21; Def. 4.2] and therefore (e) follows from [21; Theorem 4.3].

(d) $L$ is left exact as a right adjoint to the exact functor $r_{A}$. Let $f: Y_{A} \rightarrow Y_{A}^{\prime}$ be surjective. In order to prove that $L(f)$ is surjective it is sufficient to show that the restrictions of $\boldsymbol{L}(f)$ to $\left(I^{\prime}+C\right)^{*}$ and to $\left(I^{\prime \prime}\right)^{*}$ are surjective. The first is $\boldsymbol{r}_{A}(f)=f$ and the second is $r L(f): L(Y) e^{\prime \prime} \rightarrow L\left(Y^{\prime}\right) e^{\prime \prime}$. Since the induced surjection $\operatorname{soc} L(Y) e^{\prime \prime} \rightarrow \operatorname{soc} L\left(Y^{\prime}\right) e^{\prime \prime}$ splits, according to (b) $r L(f)$ is a splittable epimorphism and therefore $L(f)$ is surjective.

Since $Q^{(j)}$ and $L\left(Q_{B}^{(j)}\right)$ are indecomposable and $Q^{(j)} e^{\prime} \neq 0 \neq L\left(Q^{(j)}\right) e^{\prime}$, (e) yields $Q^{(j)} \cong L r_{A}\left(Q^{(j)}\right) \cong L\left(Q_{A}^{(j)}\right)$ because one can easily show that $r_{A} Q^{(j)}=Q_{A}^{(j)}$. The remaining statements in (d) and (c) can be proved in a similar way. The proof is complete.

In the proof of our glueing theorem we shall need the following three lemmas. We suppose that $R$ is as above.

LEMMA 5.7. Let $\tilde{Q}_{j}$ denote the sp-injective B-module $Q_{B}^{\left(c_{j}\right)}, j=0,1, \ldots, m$ (see (2.2)). Then

(a) The restriction $\widetilde{Q}_{j} e^{\prime \prime}$ of $\tilde{Q}_{j}$ to $I^{\prime \prime} \cup\{*\}$ is isomorphic to the injective envelope $E^{\prime \prime}\left(P_{*}\right)=E_{B}\left(P_{*}\right) e^{\prime \prime}$ of $P_{*}$ in $\bmod _{\mathrm{sp}}\left(e^{\prime \prime} R e^{\prime \prime}\right)$. Moreover,

$$
\left.\operatorname{dim} \tilde{Q}_{j}\right|_{c^{*}}=(0, \ldots, 0,1, \ldots, 1,1)
$$

and $\tilde{Q}_{m} \subseteq \tilde{Q}_{m-1} \subseteq \ldots \subseteq \tilde{Q}_{1} \subseteq \tilde{Q}_{0}=E_{B}\left(P_{*}\right)$ are hereditary sp-injective such that if $\operatorname{Hom}_{B}\left(\tilde{Q}_{j}, Z\right) \neq 0$ and $Z$ is indecomposable then $Z \cong \tilde{Q_{i}}$ for some $i \leqslant j$.

(b) If $Z_{B}$ is in $\bmod _{\mathrm{sp}}(B)$ and $Z e^{\prime \prime} \cong E^{\prime \prime}\left(P_{*}\right)^{h}$, where $h=\operatorname{dim}\left(Y e_{*}\right)_{F}$, then $Z_{B}$ is a direct sum of copies of hereditary sp-injective modules $\tilde{Q}_{0}, \ldots, \tilde{Q}_{m}$. In particular, $r_{B}\left(e_{j} R\right)$ has this property if $j \in I^{\prime}$.

(c) The A-modules $P_{*} \subseteq P_{c m} \subseteq \ldots \subseteq P_{c,}$ are hereditary. Moreover, $T \tilde{Q}_{m} \cong L P_{*}$ and $T \tilde{Q}_{j} \cong L P_{\mathrm{c}_{j+1}}$ for $j=0, \ldots, m-1$.

$I f$, in addition, $R$ satisfies the assumptions in Theorem 3.7 then the end of $\mathscr{Q}_{\mathrm{sp}}(B)$ and the beginning of $\mathscr{P}_{\text {up }}(A)$ have the forms $(5.8)$ below with valuations $(1,1)$ over arrows $\tilde{Q}_{j+1} \rightarrow \tilde{Q}_{j}, P_{c_{j}} \rightarrow P_{c_{j-1}}$.

Proof. (a) Note that the reflection duality (2.0) applied to the ring $B$ carries $\tilde{Q}_{j}$ to $\widetilde{B^{V}} e_{c}$, which is obviously hereditary projective because (5.0) is a splitting decomposition (look at the diagram (5.1)). Then (a) follows from the duality $D V$ and its definition. 


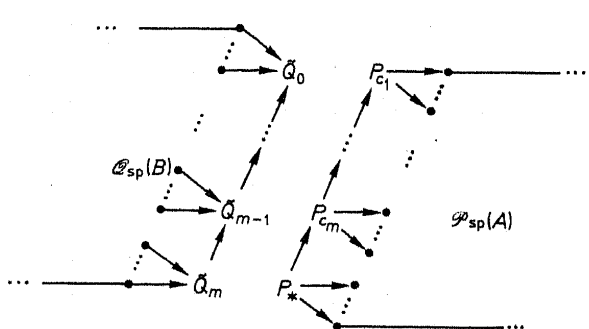

(b) It is easy to see that every indecomposable summand $Z^{\prime}$ of $Z$ has also the property of $Z$ in (b) and therefore $\operatorname{Hom}_{B}\left(\tilde{Q}_{m}, Z^{\prime}\right) \neq 0$. Since $\tilde{Q}_{m}$ is hereditary sp-injective, $Z^{\prime}$ is sp-injective and therefore there are monomorphisms $\tilde{Q}_{m} \rightarrow Z^{\prime} \rightarrow Q_{0}=E_{B}\left(P_{*}\right)$. Then (a) yields $Z^{\prime} \cong Q_{j}$ for some $j \leqslant m$.

It follows from Proposition 5.6(e) and (b) that $Y_{A}=r_{A}\left(P_{j}\right)$ is indecomposable $P_{j} \cong L Y_{A}, r_{B}\left(P_{j}\right) e^{\prime \prime} \cong Y e^{\prime \prime} \cong E^{\prime \prime}\left(P_{*}\right)^{h}$ with $h=d_{j *}$ and the proof of $(\mathrm{b})$ is complete.

(c) The first part follows from the shape of $I_{R}^{*}$ (see (5.1)) and the second one follows from (a) and the definition (3.1).

LEMMA 5.9. Let $Y, Y^{\prime}$ and $Z, Z^{\prime}$ be indecomposable modules in $\bmod _{\mathrm{sp}}(A)$ and $\bmod _{\mathrm{sp}}(B)$ respectively. Then

(a) $\operatorname{Hom}_{R}(L Y, T Z)=0$ if $Y e^{\prime} \neq 0$

(b) The natural epimorphisms

\section{(5.10) $\quad \tilde{L}: \operatorname{Irr}\left(Y, Y^{\prime}\right) \rightarrow \operatorname{Irr}\left(L Y, L Y^{\prime}\right), \quad \tilde{T}: \operatorname{Irr}\left(Z, Z^{\prime}\right) \rightarrow \operatorname{Irr}\left(T Z, T Z^{\prime}\right)$}

are bimodule isomorphisms over the natural ring isomorphisms $F(Y) \cong F(L Y)$, $F\left(Y^{\prime}\right) \cong F\left(L Y^{\prime}\right), F(Z) \cong F(T Z), F\left(Z^{\prime}\right) \cong F\left(T Z^{\prime}\right)$, respectively. In particular, $L$ and $T$ carry irreducible maps to irreducible ones.

Proof. (a) Since $Y e^{\prime} \neq 0$, by Proposition 5.6 we have $\boldsymbol{r}_{B}(L Y) e^{\prime \prime}=(L Y) e^{\prime \prime} \cong E^{\prime \prime}\left(P_{*}\right)^{h}$, where $h=\operatorname{dim}\left(Y e_{*}\right)_{F}$, and according to Lemma 5.7, $\boldsymbol{r}_{B}(L Y)$ is a direct sum of copies of $\tilde{Q}_{0}, \ldots, \tilde{Q}_{m}$. Hence if we assume that there is nonzero $f \in \operatorname{Hom}_{R}(L Y, T Z)$ then the restriction of $f$ to $\operatorname{soc} L Y \subseteq r_{B}(L Y) e^{\prime \prime}$ is nonzero and therefore $\operatorname{Hom}_{B}\left(\tilde{Q}_{j}, Z\right) \neq 0$. It follows from Lemma 5.7 that $Z \cong \tilde{Q}_{i}$ for some $0 \leqslant i \leqslant j$ and $T Z \cong T \tilde{Q}_{j} \cong L P_{c_{j+1}}$, where $P_{c_{m+1}}=P_{*}$. Hence $\operatorname{Hom}_{R}(L Y, T Z) \cong \operatorname{Hom}_{R}\left(L Y, L P_{c_{j+1}}\right) \cong \operatorname{Hom}_{A}\left(Y, P_{c_{j+1}}\right)=0$, because $P_{c_{j+1}}$ is hereditary projective and $Y \nsupseteq P_{c_{i}}$ for $i=1, \ldots, m+1$. This contradiction finishes the proof of (a).

(b) Suppose $\tilde{T}(\tilde{g})=0$, where $g \in J\left(Z, Z^{\prime}\right)$. Then $T(g)$ has a factorization $T Z \stackrel{h}{\rightarrow} X \stackrel{t}{\rightarrow} T Z^{\prime}$ in $\bmod _{\mathrm{sp}}(R)$ with $h \in J(T Z, X), t \in J\left(X, T Z^{\prime}\right)$ and in view of (a), $X$ can be chosen of the form $X=T\left(Z^{\prime \prime}\right)$. Since $T$ is full and faithful, $g=t h \in J^{2}\left(Z, Z^{\prime}\right)$. Thus $\bar{g}=0$ and $\tilde{T}$ is bijective.

Suppose $\tilde{L}(\bar{f})=0$ where $f \in J\left(Y, Y^{\prime}\right)$. If $L(Y)$ is not in the image of $T$ then above we conclude that $\bar{f}=0$. Suppose $L(Y) \cong T(Z)$. Note that this happens if and only if $Y \cong P_{c_{j}}$ for some $j=1, \ldots, m+1, c_{m+1}=*$. Suppose $Y=P_{c_{j}}$ and note that $L(Y) \cong T\left(\tilde{Q}_{j-1}\right)$ (Lemma 5.7). If $L Y^{\prime}$ is in the image of $T$ then we are done by (5.9) for $T$. Suppose $L Y^{\prime} \notin \operatorname{Im} T$ and $\tilde{L}(\bar{f})=0$. Then $L(f)$ is a sum of composed maps $L P_{c_{j}} \cong T \tilde{Q}_{j-1} \stackrel{h_{s}}{\rightarrow} X_{s} \stackrel{t_{s}}{\rightarrow} L Y^{\prime}$ where $h_{s} \in J\left(T \tilde{Q}_{j-1}, X_{s}\right), t_{s} \in J\left(X_{s}, L Y^{\prime}\right)$ are both nonzero. Assume that $X_{s} \cong T\left(Z_{s}\right)$. Then $h_{s}=\boldsymbol{T}\left(h_{s}^{\prime}\right)$ for some $h_{s}^{\prime}: \tilde{Q}_{j-1} \rightarrow Z_{s}$ and Lemma 5.8 yields $Z_{s} \cong \tilde{Q}_{i}, X_{s} \cong T\left(Z_{s}\right) \cong L P_{c_{i+1}}$ for some $i \leqslant j-1$. This proves that all modules $X_{s}$ are of the form $L\left(Y_{s}\right)$ because of Proposition 5.6(e). It follows that $f=\sum f_{s}^{\prime} f_{s}^{\prime \prime}$ for some $f_{s}^{\prime} \in J\left(Y_{s}, Y^{\prime}\right), f_{s}^{\prime \prime} \in J\left(P_{c_{j}}, Y_{s}\right)$ and therefore $\bar{f}=0$. This finishes the proof.

LEMma 5.11. Suppose that $Y_{A}, Z_{B}$ are indecomposable and let

$$
\text { V): } 0 \rightarrow Y^{\prime \prime} Y^{\prime} \rightarrow Y^{\prime \prime} \rightarrow 0, \quad 3: 0 \rightarrow Z \stackrel{\circ}{\rightarrow} Z^{\prime} \rightarrow Z^{\prime \prime} \rightarrow 0
$$

be almost split exact sequences in $\bmod _{\mathrm{sp}}(A)$ and $\bmod _{\mathrm{sp}}(B)$ respectively. Then $L \mathfrak{Y}, T 3$ are almost split sequences.

Proof. Since $L Y, L Y^{\prime \prime}, T Z, T Z^{\prime \prime}$ are indecomposable it is sufficient to prove that $L(u), T(v)$ are left almost split. Let $f: L Y \rightarrow X$ be a nonzero nonisomorphism and let $X$ be indecomposable in $\bmod _{\text {sp }}(R)$. We know from Proposition $5.6(\mathrm{e})$ that either $X \cong L U$ or $X \cong T V$ for some $U$ in $\bmod _{\mathrm{sp}}(A), V$ in $\bmod _{\mathrm{sp}}(B)$. If $X \cong \boldsymbol{L} U$ there is a nonisomorphism $g: Y \rightarrow U$ such that $f=L(g)$. Since $\mathfrak{Y}$ is almost split $g$ factorizes through $u$ and $f$ factorizes through $L(u)$. Suppose $X \cong T V$. Since $f \neq 0$, in view of Lemma 5.9 (a) we have $(\boldsymbol{L} Y) e^{\prime}=0$ and therefore $\boldsymbol{L Y} \cong \boldsymbol{T Z}$ for some $Z$. It follows as in the proof above that $Y \cong P_{c j}, Z \cong \tilde{Q}_{j-1}$ for some $j=1, \ldots, m+1$ (see 5.8). Since $\operatorname{Hom}_{B}\left(\tilde{Q}_{j-1}, V\right) \cong \operatorname{Hom}_{R}(L Y, X) \neq 0$, in view of Lemma 5.7 we have $V \cong \widetilde{Q_{i}}$ for some $i<j$ and $X \cong T V \cong T \tilde{Q}_{i} \cong L P_{c_{i+1}}$. If $h: Y \rightarrow P_{c_{i+1}}$ is such that $L(h)=f$ then $h$ factorizes through $u$ and $f$ factorizes through $L(u)$ as required. Consequently $L \mathfrak{Y}$ is almost split. The proof of the remaining part is left to the reader.

Now we are able to prove the main result of this section.

THEOREM 5.12. Let $R$ be an artinian right peak PI-ring of the form (1.1). Suppose that $R$ is upper triangular, has almost split sp-sequences and that $\left(I_{R}^{*}, d\right)$ is a valued poset with respect to $i \prec j \Leftrightarrow i M_{j} \neq 0$. If $\left(I_{R}^{*}, d\right)$ has a splitting decomposition (5.0) then

(a) The exact functors $\boldsymbol{L}, \boldsymbol{T}(5.4)$ carry almost split sequences to almost split sequences and induce the bimodule isomorphisms (5.10)

(b) Every almost split sequence $\mathfrak{X}$ in $\bmod _{\mathrm{sp}}(R)$ is either of the form $T(3)$ or of the form $L(\mathfrak{Y})$, where 3 and 9 ) are almost split sequences. Every irreducible map $f: X \rightarrow X^{\prime}$ between indecomposable modules in $\bmod _{\mathrm{sp}}(R)$ is of one of the the forms $\boldsymbol{L}(g), \boldsymbol{T}(h)$, where $g$ and $h$ are irreducible.

(c) An indecomposable module $X$ in $\bmod _{\mathrm{sp}}(R)$ belongs to $\operatorname{Im} L \cap \operatorname{Im} T$ if and only if $X \cong L\left(P_{c_{j}}\right) \cong T\left(\tilde{Q}_{J-1}\right)$ for some $j=1, \ldots, m+1$, where $c_{m+1}=*$ (see 5.8$)$.

(d) If, in addition, $R$ satisfies the assumptions of Theorem 3.7 then $\left(\Gamma_{\mathrm{sp}}(R), d, \Delta, \chi\right)$ has the form below (in the notation of Remark 3.12) obtained from $\left(\Gamma_{\mathrm{sp}}(B), \boldsymbol{d}, \Delta, \chi\right)$ and $\left(\Gamma_{\mathrm{sp}}(A), d, \Delta, \chi\right)$ by the identification of the final section $\tilde{Q}_{m} \rightarrow \tilde{Q}_{m-1} \rightarrow \ldots \rightarrow \tilde{Q}_{0}$ in $\mathscr{Q}_{\mathrm{sp}}(B)$ with the starting section $P_{*} \rightarrow P_{c_{m}} \rightarrow \ldots \rightarrow P_{c_{1}}$ of $\mathscr{P}_{\text {sp }}(A)$ (see 5.8), where $A, B$ are the rings (5.3) and there are no maps from the right to the left. 


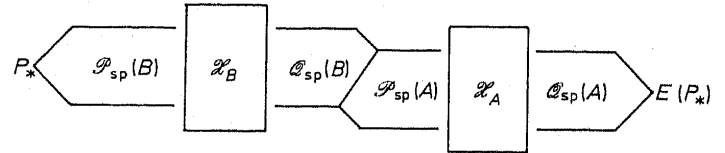

Proof. (a) follows from Lemmas 5.9 and 5.11, whereas (c) follows from the proof of Lemma 5.9.

(b) We know from Proposition 5.6 (e) that every indecomposable module $X$ in $\bmod _{\text {sp }}(R)$ belongs either to $\operatorname{Im} T$ or to $\operatorname{Im} L$. Let

$$
\mathfrak{x}: 0 \rightarrow X \rightarrow X^{\prime} \rightarrow X^{\prime \prime} \rightarrow 0
$$

be an almost split sequence in $\bmod _{\mathrm{sp}}(R)$ and let $X$ be indecomposable. If $X \cong L Y$ then we know from Proposition 5.6(d) that $Y$ is not sp-injective and therefore there is an almost split sequence $\mathfrak{Y}$ in $\bmod _{\mathrm{sp}}(A)$ starting with $Y$. By Lemma $\left.5.11, L \mathfrak{V}\right)$ is an almost split sequence starting with $X$ and therefore, $\mathfrak{X} \cong L \mathfrak{2}$.

Suppose now that $X \notin \operatorname{Im} L$. Then $X \cong T Z$ for some $Z$ and we know from (c) that $X \supsetneqq T\left(\widetilde{Q}_{j}\right)$ for $j=0,1, \ldots, m$. Then, in view of Proposition 5.6 (c), $Z$ is not sp-injective and therefore there is an almost split sequence $\mathbb{R}$ in $\bmod _{\mathrm{sp}}(B)$ starting with $Z$. It follows from Lemma 5.11 that $\mathfrak{X} \cong T(3)$ as desired. Since the second statement in (b) follows from the first, (b) is proved.

Since (d) follows from (a)-(c) the theorem is proved

COROLlARY 5.13. Let I be a poset having a disjoint union poset decomposition $I=I^{\prime}+C+I^{\prime \prime}$, where $C: c_{1} \rightarrow c_{2} \rightarrow \ldots \rightarrow c_{m}$ is a chain, $I^{\prime}, I^{\prime \prime}$ are nonempty and the condition (i) below (5.0) is satisfied. If $\boldsymbol{T}:\left(C+I^{\prime \prime}\right)$-sp $\rightarrow I$-sp is the natural embedding functor and $L:\left(I^{\prime}+C\right)$-sp $\rightarrow I$-sp is given by $L\left(M, M_{j}\right)_{j \in I^{\prime}+C}=\left(M, \hat{M}_{i}\right)_{i \in I}$, where $\hat{M}_{i}=M_{i}$
for $i \in I^{\prime}+C$ and $\hat{M}_{i}=M$ for $i \in I^{\prime \prime}$, then $T, L$ are exact and have the properties (a)-(d) of Theorem 5.12 with $I$-sp, $\left(I^{\prime}+C\right)$-sp, $\left(C+I^{\prime \prime}\right)$-sp and $\bmod _{\mathrm{sp}}(R), \bmod _{\mathrm{sp}}(A), \bmod _{\mathrm{sp}}(B)$ interchanged.

Proof. Apply Theorem 5.12 to $R=F I^{*}$. Note that $\bmod _{\mathrm{sp}}\left(F I^{*}\right) \cong I$-sp, $\bmod _{\mathrm{sp}}(A) \cong\left(I^{\prime}+C\right)$-sp, $\bmod _{\mathrm{sp}}(B) \cong\left(C+I^{\prime \prime}\right)$-sp and the functors $L, T$ above coincide with the functors $L, T$ in (5.4) (see [30]).

Remark 5.14. (1) Let

$$
R=\left[\begin{array}{cc}
S & { }_{S} M_{B} \\
0 & B
\end{array}\right]
$$

where $S=e^{\prime} R e^{\prime}, M=e^{\prime} R \eta$ (see 5.2). Then $M_{B}$ is a direct sum of copies of hereditary sp-injective $B$-modules $\widetilde{Q}_{0}, \ldots, \widetilde{Q}_{m}$ (Lemma $\left.5.7(\mathrm{~b})\right)$ and therefore we are in a situation similar to that in [34] (compare Theorem 5.12 with [34; Theorem 1 and Remark 1])

(2) It would be interesting to prove a counterpart of Theorem 5.12 for a ring splitting of $R$ in the sense of [21; Definition 4.2]. Example 4.4 shows that Theorem 5.12 and Lemmas preceding it do not hold for ring splittings. In fact $I_{T}=I^{\prime}+C+I^{\prime \prime}$ with $I^{\prime}=\{1,2,4\}, C=\{3,6\}, I^{\prime \prime}=\{5\}$ induce a splitting system of functors (5.4) with $R=T$. The modules from $\operatorname{Im} L$ and from $\operatorname{Im} L \cap \operatorname{Im} T$ in Figure 1 are underlined and wave underlined respectively.
Another illustration of the construction $\left(\Gamma_{\text {ap }}(R), d, \Delta\right)$ in the case of a ring splitting by successively applying (5.4) is given in Example 5.15 below.

(3) In [33] a splitting theorem is proved for directed multipeak bound quiver algebras It plays an important role in determining coordinate supports of indecomposable socle projective modules over covering algebras of right peak algebras (see [32])

ExAmple 5.15. Let $\boldsymbol{C}$ and $\boldsymbol{R}$ be the complex and real numbers, respectively, and

$$
R=\left[\begin{array}{llllllll}
C & 0 & 0 & C & C & C & C \\
& R & R & 0 & C & C & C \\
& & R & 0 & 0 & R & C \\
& & & C & C & 0 & C \\
& & & & C & 0 & C \\
& & & & & R & C \\
0 & & & & & & C
\end{array}\right]
$$

where $c_{236}: R \otimes R \rightarrow C$ is the natural embedding, $c_{26 *}: C \otimes C \rightarrow C$ is such that the composed map $\bar{c}_{26 *}: C \rightarrow \operatorname{Hom}_{C}(C, C) \cong C$ is the identity. The remaining maps $c_{i j k}$ are multiplications. Note that

$\left(I_{R}^{*}, d\right)$

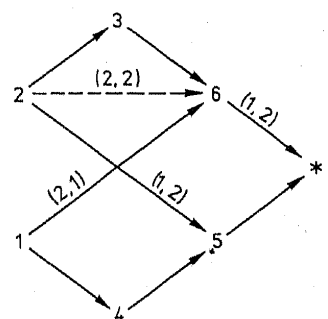

has no splitting decomposition; however, $I_{R}=I^{\prime}+C^{\prime}+I^{\prime \prime}$ with $I^{\prime}=\{1\}, C=\{2,3,4,5\}$, $I^{\prime \prime}=\{6\}$ induces a ring splitting with the corresponding rings $A, B$ as in (5.3) obtained from $R$ by omitting the first row and the first column, and the sixth row and the sixth column respectively. Note that $I_{A}=\{1,2,3,4,5\}, I_{B}=\{2,3,4,5,6\}$. For the rings $A$ and $B$ there exist ring splittings induced by the following decompositions:

$$
I_{A}=I_{A}^{\prime}+C_{A}+I_{A}^{\prime \prime} \text { and } I_{B}=I_{B}^{\prime}+C_{B}+I_{B}^{\prime \prime} \text {, }
$$

where $I_{A}^{\prime}=\{2\}, C_{A}=\{1,3,4\}, I_{A}^{\prime \prime}=\{5\}, I_{B}^{\prime}=\{2\}, C_{B}=\{3,4,6\}, I_{B}^{\prime \prime}=\{5\}$.

Let $\left(A^{1}, B^{1}\right)$ and $\left(A^{2}, B^{2}\right)$ be the corresponding splitting pairs like in (5.3). Then

$$
\left(I_{A^{1}}^{*}, d\right): 2 \rightarrow 3 \stackrel{(1,2)}{\longrightarrow} * \leftarrow 4-4 \leftarrow 1, \quad\left(I_{B^{*}}^{*}, d\right): 1 \rightarrow 4 \rightarrow 5 \rightarrow * \stackrel{(2,1)}{\longleftarrow} 2,
$$

$\left(l_{A^{2}}^{*}, d\right):$

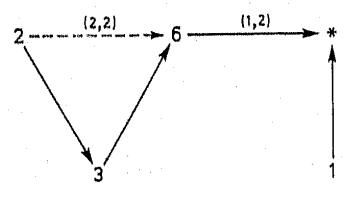

$\left(I_{B^{1}}^{*}, d\right): 3 \rightarrow 6 \stackrel{(1,2)}{\longrightarrow} * \leftarrow 5 \leftarrow 4$. 
Note that the rings $A^{1}$ and $B^{2}$ are hereditary sp-representation-infinite of type $\widetilde{\boldsymbol{F}}_{42}^{\prime}$. The ring $B^{1}$ is hereditary representation-finite of type $\boldsymbol{B}_{5}^{\prime \prime}$ and the ring $A^{2}$ is sp-representation-finite of type $\hat{\boldsymbol{F}}_{4}^{\prime}$ (see [21; Appendix]). The Auslander-Reiten valued quivers of the rings above are presented in Figure 2 .
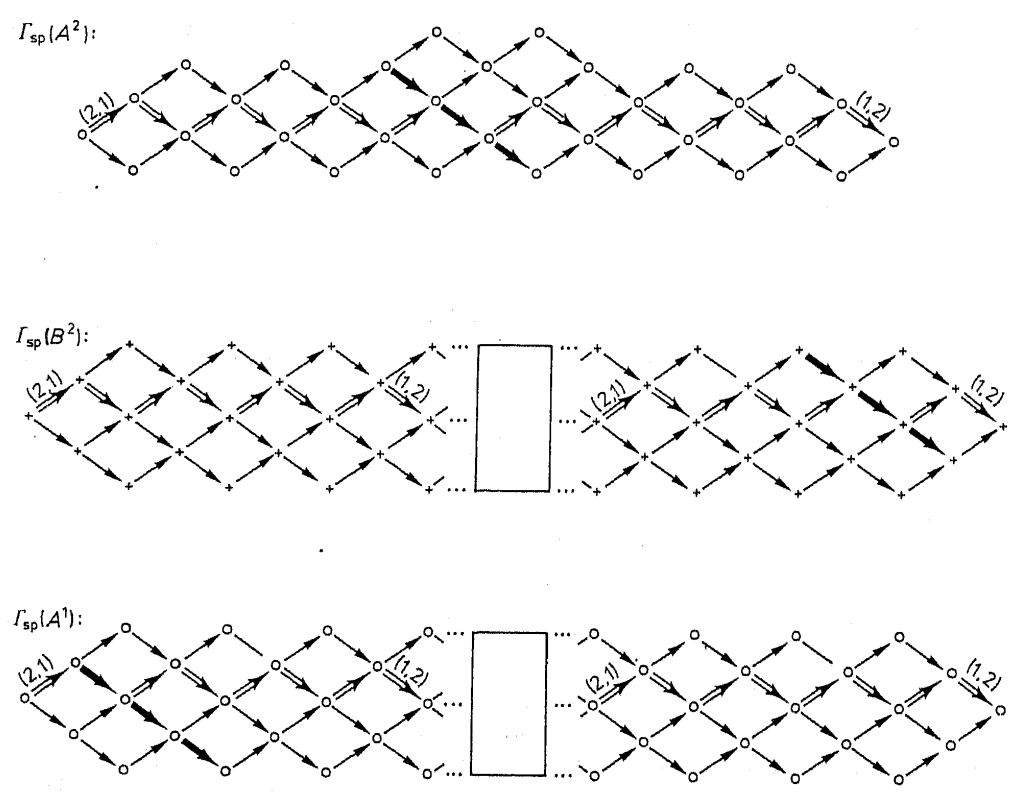

$\Gamma_{\mathrm{sp}}\left(B^{1}\right):$

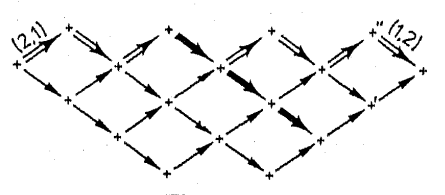

Fig. 2

Let us denote by $L, T, L^{1}, T^{1}, L^{2}, T^{2}$ the functors (5.4) corresponding to the pairs $(A, B)$, $\left(A^{1}, B^{1}\right),\left(A^{2}, B^{2}\right)$, respectively. One can show that $\Gamma_{\mathrm{sp}}(A)$ and $\Gamma_{\mathrm{sp}}(B)$ are obtained from $\Gamma_{\mathrm{sp}}\left(A^{1}\right), \Gamma_{\mathrm{sp}}\left(B^{1}\right)$ and $\Gamma_{\mathrm{sp}}\left(A^{2}\right), \Gamma_{\mathrm{sp}}\left(B^{2}\right)$ by glueings along sections in $\operatorname{Im} \boldsymbol{L}^{1} \cap \operatorname{Im} \boldsymbol{T}^{1}$ and $\operatorname{Im} L^{2} \cap \operatorname{Im} T^{2}$ presented in Figure 3. The marked sections represent the indecomposables

$(0,0,1,0,1,1) \stackrel{(1,2)}{\longrightarrow}(0,0,2,1,2,2) \rightarrow(1,0,2,1,2,2) \rightarrow(0,0,2,0,1,1)$,

$(0,1,0,1,2,1) \rightarrow(0,2,1,2,3,2) \stackrel{(1,2)}{\longrightarrow}(0,2,1,2,4,2) \rightarrow(0,2,0,1,2,1)$
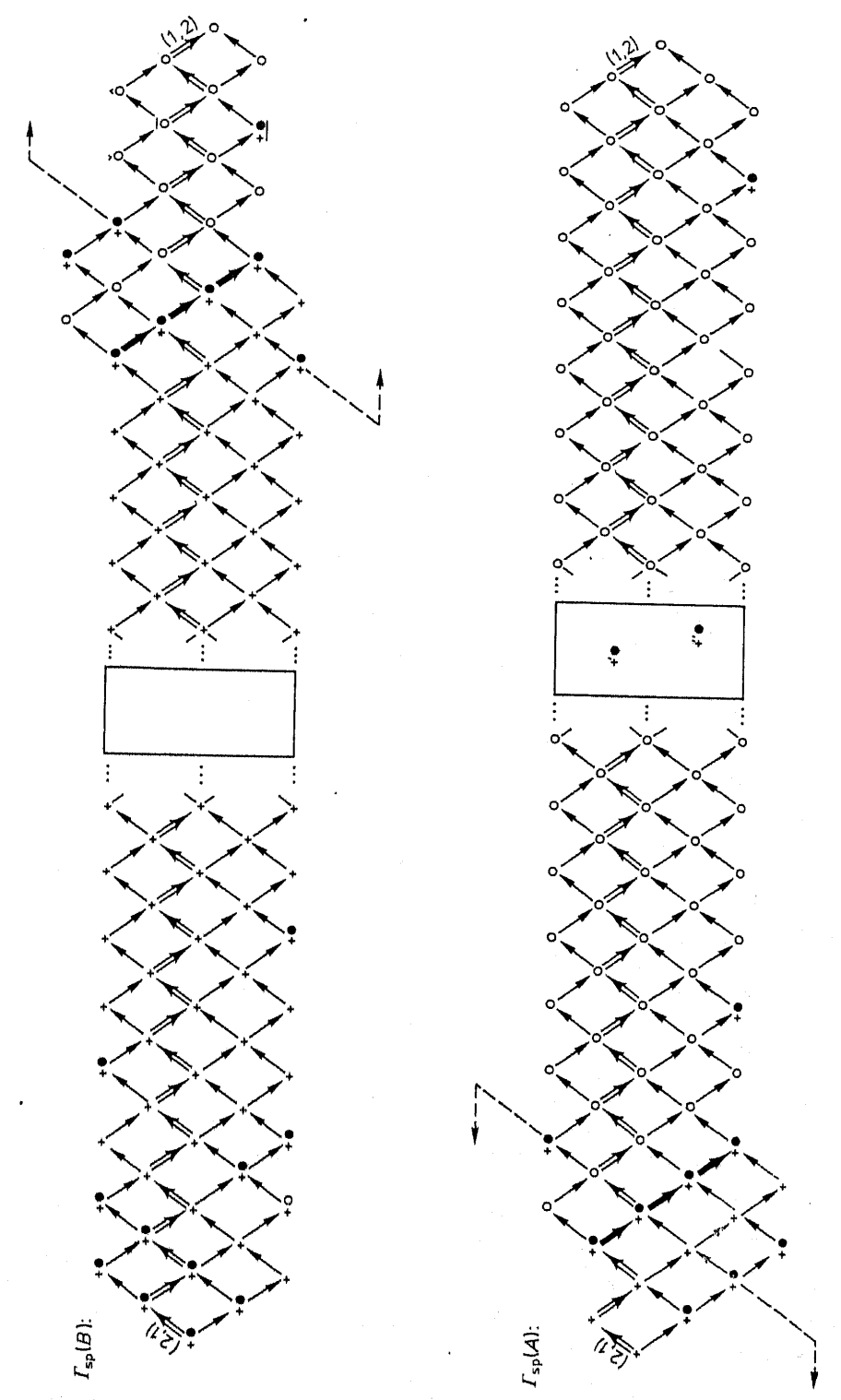
in terms of dimension vectors $\left(x_{1}, \ldots, x_{5}, x_{*}\right),\left(x_{2}, \ldots, x_{6}, x_{*}\right)$, respectively. Moreover $+" \bullet$ and $++^{\prime}$ are the vectors $(1,0,1,1,1,1)$ and $(0,0,2,1,1,1) ;+^{\prime \prime} \bullet$ belongs to a tube of rank 3 and t' belongs to a tube of rank 2 .

We mark by $\circ$ the modules in $\operatorname{Im} L, \cdot \operatorname{Im} L^{1}, \operatorname{Im} L^{2}$, and by + the ones in $T, \operatorname{Im} T^{1}, \operatorname{Im} T^{2}$. The modules in $\operatorname{Im} T^{1} \cap \operatorname{Im} L^{1}, \operatorname{Im} T^{2} \cap \operatorname{Im} L^{2}, \operatorname{Im} T \cap \operatorname{Im} L$ are denoted by $+\bullet$. The glueing respects the natural order in the quivers.

Now by simple calculations one can show that $\Gamma_{\mathrm{sp}}(R)$ can be obtained from $\Gamma_{\mathrm{sp}}(A)$ and $\Gamma_{\mathrm{sp}}(B)$ by glueing along a section in $\operatorname{Im} L \cap \operatorname{Im} T$ as presented in Figure 4. The indecomposable modules in this section are represented in terms of their dimension

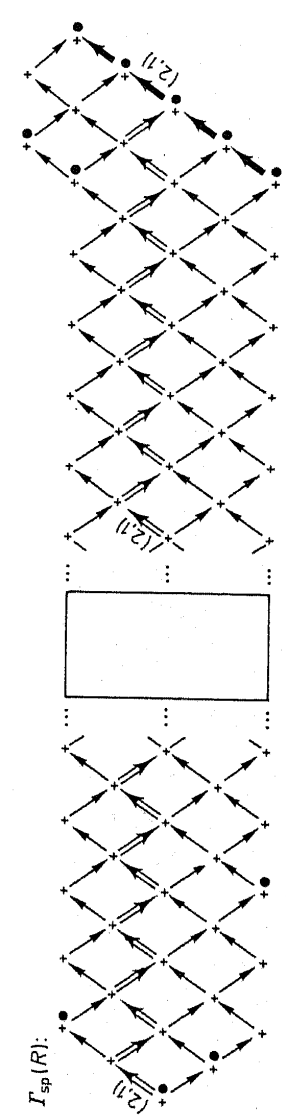
vectors $\left(x_{1}, x_{2}, \ldots, x_{5}, x_{6}, x_{*}\right)$, i.e.: $(0,0,0,1,1,2,1) \rightarrow(0,0,2,1,1,4,2) \rightarrow(0,0,2,1$, $2,4,2) \stackrel{(2,1)}{\longrightarrow}(0,1,2,1,2,4,2) \rightarrow(0,0,1,1,1,2,1)$. The singular point $+^{\prime} \bullet=+\bullet$ in the square belongs to a tube of rank 2 , its dimension vector is $(0,0,2,1,1,2,1)$. Note that the triple

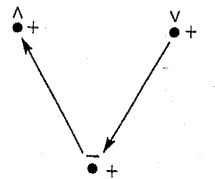

in the square equals $(1,0,2,0,1,2,1) \rightarrow(2,0,3,1,2,4,2) \rightarrow(1,0,1,1,1,2,1)$ and belongs to a tube of rank 3 . The modules $+\hat{\mathbf{\theta}},+\check{\mathbf{\varphi}},+$ "⿺ form one $\Delta$-orbit in the tube The boldface sections in Figure 4 should be identified.

\section{References}

[1] M. Auslander, Representation theory of artin algebras II, Comm. Algebra 1 (1974), 269-310.

[2] - Applications of morphisms determined by objects, in: Proc. of the Philadelphia Conf., Lecture Notes in Pure and Appl. Math. 37 Dekker, New York 1978, 245-327.

[3] M. A uslander and I. Reiten, Representation theory of Artin algebras III, Comm. Algebra 3 (1975), 269-310.

[4] M. Auslander and S. O. Smal $\phi$, Almost split seguences in subcategories, J. Algebra 69 (1981), $426-454$

[5] R. Bautista, Torsion theories and Auslander-Reiten sequences, An. Inst. Mat. Univ. Nac. Autónoma México 19 (1) (1979), 1-19.

[6] - Sections in Auslander Reiten quivers, in: Proc. ICRA Il (Ottawa 1979), Lecture Notes in Math. 832, Springer 1980, 74 96.

[7] R. Bautista and F. Larrion, Auslander-Reiten quivers for certain algebras of finite representation type, J. London Math. Soc. (2) 26 (1) (1982), 43-52.

[8] R. Bautista, F. Larrion and L. Salmeron, On simply connected algebras, J. London Math. Soc. (2) 27 (2) (1983), 212 220

[9] R. Bautista and R. Martinez-Villa, Representations of partially ordered sets and 1-Gorenstein algebras, in: Proc. Antwerp Conf. (Antwerp 1978), Lecture Notes in Pure and Appl. Math. 57, Dekker, New York 1979, 385-433.

[10] K. Bongartz and P. Gabriel, Covering spaces in representation theory, Invent. Math. 65 (1982), $331 \cdots 378$. 
[11] D. Bünermann, Auslander-Reiten quivers of exact one-parameter partially ordered sets, in: Proc. ICRA III (Puebla 1980), Lecture Notes in Math. 903, Springer, 1981, 55-61.

[12] P. Dowbor, Representations of hereditary rings (in Polish), Ph. D. thesis, Nicholas Copernicus Univ., Torun 1981.

[13] P. Dowbor and D. Simson, Quasi-Artin species and rings of finite representation type, J. Algebra 63 (1980), 435-443.

[14] P. Gabriel, Auslander-Reiten sequences and representation-finite algebras, Proc. ICRA II (Ottawa 1979), Lecture Notes in Math. 831, Springer, 1981, 1-71.

[15] - The universal cover of a representation finite algebra, Proc. ICRA III (Puebla 1980), Lecture Notes in Math. 903, Springer, 1981, 68-105.

[16] D. Happel, Composition factors for indecomposable modules, Proc. Amer. Math. Soc. 86 (1) (1982), 29-31.

[17] D. Happel and C. M. Ringel, Tilted algebras, Trans. Amer. Math. Soc. 274 (2) (1982), $399-443$

[18] K. Igusa and G. Todorov, Radical layers of representable functors, J. Algebra 89 (1984), 105-147.

[19] - - A characterization of finite Auslander-Reiten quivers, J. Algebra 89 (1984), 148-177.

[20] B. Klemp and D. Simson, A diagrammatic characterization of schurian vector space PI-categories of finite type, Bull. Polish Acad. Sci. Math. 32 (1984), 11-18.

[21] - - Schurian sp-representation-finite right peak PI-rings and their indecomposable socle projective modules, J. Algebra, 1990, in print.

[22] L. A. Nazarova and A. V. Roiter, Representations of partially ordered sets, Zap. Nauchn. Sem. Leningrad. Otdel. Mat. Inst. Steklov. (LOMI) 28 (1972), 5-31 (in Russian)

[23] C. M. Ringel, On algorithms for solving vector space problems. I. Report on the Brauer-Thrall conjectures: Roiter's theorem and the theorem of Nazarova and Roiter, in: Proc.
Workshop ICRA II (Ottawa 1979), Lecture Notes in Math. 831, Springer, 1980, 104-136.

[24] - Kawada's theorem, Abelian group theory, Lecture Notes in Math. 874, Springer, 1981, 431-447.

[25] - Tame algebras and integral quadratic forms, Lecture Notes in Math. 1099, Springer, 1984.

[26] K. W. R oggen kam p, Indecomposable representations of orders, in: Topics in Algebra, Part I: Rings and Representations of Algebras, Banach Center Publications 26, PWN, Warszawa I: Rings and Representations of Algebras, Banach Center Publications 26, PWN, Warszawa 1990, 449-491.

[27] D. Sim s on, Partial Coxeter functors and right pure semisimple hereditary rings, J. Algebra 71 (1981), 195-218.

[28] - Vector space categories, right peak rings and their socle projective modules, J. Algebra 92 (1985), 532-571

[29] - Socle reductions and socle projective modules, J. Algebra 103 (1986), 18-68.

[30] - On Representations of Partially Ordered Sets, Paderborn, July 1986, $188 \mathrm{p}$

[31] - A narrow over-ring adjustification functor, J. Algebra, to appear.

[32] - Representations of bounded stratified posets, coverings and socle projective modules, in: Topics in Algebra, Part I: Rings and Representations of Algebras, Banach Center Publications 26. PWN, Warszawa 1990, 499-453.

[33] - A splitting theorem for multipeak path algebras, Fund. Math. 137 (2) (1990).

[34] D. Simson and A. Skowroński, Extensions of artinian rings by hereditary injective modules, in: Proc. ICRA III (Puebla 1980), Lecture Notes in Math. 903 (1981), 315 -330.

INSTITUTE OF MATHEMATICS

NICHOLAS COPERNICUS UNIVERSITY

ul. Chopina $12 / 18$

87-100 Torun, Poland

\section{On supercomplete uniform spaces IV Countable products}

by

Aarno Hohti (Helsinki) and Jan Pelant (Praha)

Abstract. We show that the product of countably many supercomplete $C$-scattered spaces is supercomplete. The result implies similar but weaker theorems of [1], [17] and [4]

1. Introduction. It is well known that the product of paracompact spaces is in general not paracompact. It was proved by Z. Frolik in [5] that a countable product of locally compact paracompact spaces is paracompact. The same is true for the larger class of paracompact $p$-spaces of Arkhangel'skii [2]. Recently a weaker structural condition of being scattered or $C$-scattered has been used by K. Alster [1], M. E. Rudin and S. Watson [17], and by L. M. Friedler, H. W. Martin and S. W. Williams in [4], to obtain similar results. We prove in this paper a natural extension of their results by showing that a countable product of supercomplete $C$-scattered spaces is supercomplete. The notion of supercompleteness was defined by J. R. Isbell in [13]; by his result - we can take it as a definition - a uniform space $\mu X$ is supercomplete iff $X$ is topologically paracompact and the Ginsburg-Isbell locally fine coreflection ([6]) $\lambda \mu$ is the fine uniformity of $\mathscr{F}(X)$ of $X$. By using the concept of metric-fine coreflections, we show at the end of the paper that a countable product of $\sigma$-C-scattered paracompact spaces is paracompact.

Our proof uses a simple recursive technique based on well-founded (or Noetherian) trees, applied e.g. in [11], [12], [15] in the context of uniform spaces.

2. Preliminaries. This section consists of preliminary definitions. We refer the reader to [14] for basic information on uniform spaces. For the definition of the Ginsburg-Isbell locally fine coreflection $\lambda$, the reader is referred to the first three papers [8], $[9],[10]$ in our study on supercomplete spaces. A well-founded tree is a partially ordered set $\mathscr{T}=(T, \leqslant)$ with a unique minimal element $\operatorname{Root}(\mathscr{T})$ such that every branch, i.e., maximal linearly ordered subset, of $\mathscr{T}$ is finite. We denote by End $(\mathscr{T})$ the set of all $\leqslant$-maximal elements of $\mathscr{T}$. Given $p \in T$, the set of all immediate $\leqslant$-successors of $p$ is denoted by $S(p)$. Thus, $S(p)=\{q \in T: q>p$ and $q>r>p$ for no $r \in T\}$. Furthermore, 